\title{
IL-23R-activated STAT3/STAT4 is essential for Th1/Th17-mediated CNS autoimmunity
}

Priscilla W. Lee, ${ }^{1,2}$ Alan J. Smith, ${ }^{3}$ Yuhong Yang, ${ }^{4}$ Amanda J. Selhorst, ${ }^{1}$ Yue Liu, ${ }^{1}$ Michael K. Racke, ${ }^{4,5}$ and Amy E. Lovett-Racke ${ }^{1,5}$

'Department of Microbial Infection and Immunity, ${ }^{2}$ Molecular, Cellular and Developmental Biology Program, ${ }^{3}$ Medical Scientist Training Program, ${ }^{4}$ Department of Neurology, and ${ }^{5}$ Department of Neuroscience, The Ohio State University Wexner Medical Center, The Ohio State University, Columbus, Ohio, USA.

The factors that promote the differentiation of pathogenic T cells in autoimmune diseases are poorly defined. Use of genetically modified mice has provided insight into molecules necessary for the development of autoimmunity, but the sum of the data has led to contradictory observations based on what is currently known about specific molecules in specific signaling pathways. To define the minimum signals required for development of encephalitogenic $T$ cells that cause CNS autoimmunity, myelin-specific T cells were differentiated with various cytokine cocktails, and pathogenicity was determined by transfer into mice. IL-6+IL-23 or IL-12+IL-23 generated encephalitogenic $T$ cells and recapitulated the essential cytokine signals provided by antigenpresenting cells, and both IL-6 and IL-12 induced IL-23 receptor expression on both mouse and human naive T cells. IL-23 signaled through both STAT3 and STAT4, and disruption in STAT4 signaling impaired CNS autoimmunity independent of IL-12. These data explain why IL-12-deficient mice develop CNS autoimmunity, while STAT4-deficient mice are resistant. CD4+ memory T cells from multiple sclerosis patients had significantly higher levels of p-STAT3/p-STAT4, and p-STAT3/ p-STAT4 heterodimers were observed upon IL-23 signaling, suggesting that $p-S T A T 3 / p-S T A T 4$ induced by IL-23 signaling orchestrate the generation of pathogenic T cells in CNS autoimmunity, regardless of Th1 or Th17 phenotype.

Conflict of interest: The authors have declared that no conflict of interest exists.

Submitted: November 7, 2016 Accepted: July 27, 2017 Published: September 7, 2017

\section{Reference information:} JCI Insight. 2017;2(17):e91663. https://doi.org/10.1172/jci. insight. 91663.

\section{Introduction}

While the cause of multiple sclerosis (MS) remains unknown, it is thought to be mediated by myelin-reactive $\mathrm{CD}^{+} \mathrm{T}$ cells (1). However, not all myelin-reactive $\mathrm{T}$ cells are encephalitogenic, illustrating that myelin specificity is not sufficient for T cells to cause MS. In fact, the frequencies of myelin-reactive T cells in the periphery are comparable between MS patients and healthy individuals, but these T cells have an effector phenotype in patients with MS (2-4), indicating that these T cells have previously encountered antigen (Ag) and gained encephalitogenicity. During primary differentiation in the periphery, antigen-presenting cells (APC) present Ag and secrete cytokines that provide unique microenvironments for naive T cells and initially decide the fate of $\mathrm{T}$ cell phenotypes.

Both Th1- and Th17-mediated pathogenic mechanisms have been proposed in MS (5). In experimental autoimmune encephalomyelitis (EAE), an animal model of MS, Th1 cells were initially found capable of inducing disease by adoptive transfer studies (6-9), suggesting a dominant role of Th1-mediated pathogenesis. The observation that myelin-reactive T cells in MS patients frequently had a Th1 phenotype $(1,10-13)$ led to significant efforts investigating the therapeutic potential of Th1-associated molecules. The experiments on systemic neutralization or genetic deletion of IFN- $\gamma$ showed that this Th1 signature cytokine was not required for EAE induction (2-4, 14-17). Despite the crucial role of IL-12 in promoting Th1 differentiation, deletion of the IL-12-specific subunit (IL-12-p35) in mice did not ameliorate EAE susceptibility $(5,18,19)$. In contrast, loss of STAT4, which transduces signals distal to the IL-12 receptor, made mice resistant to EAE $(6-9,20)$. This suggested that STAT4 was associated with encephalitogenicity independent of IL-12 signaling. More recently, IL-23, a cytokine sharing the IL-12p40 molecule with IL-12, was identified as an absolutely required element in EAE development (21). IL-23 expanded the 
A

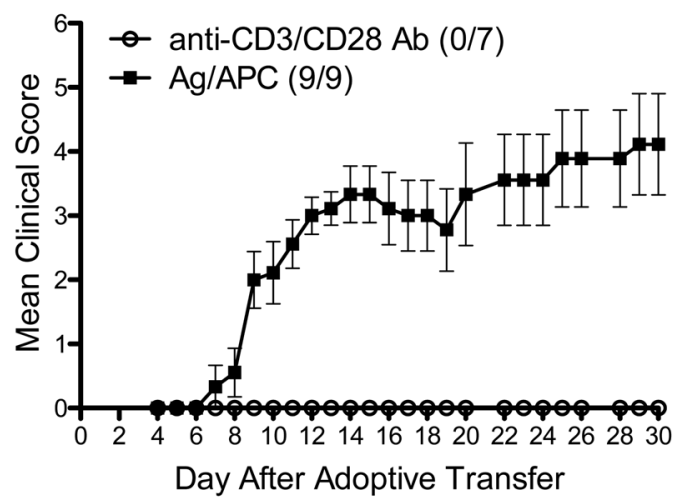

B

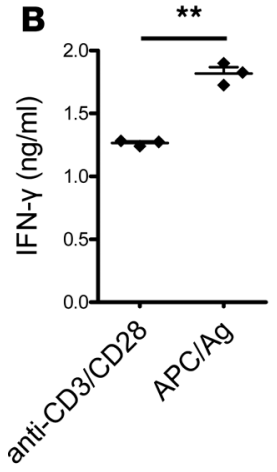

Figure 1. MBP-specific $T$ cells activated with anti-CD3/28 fail to induce EAE. Splenocytes from naive V $\alpha 2.3 / \mathrm{V} \beta 8.2$ TCR Tg mice were activated in vitro with anti-CD3/CD28 or myelin basic protein Ac1-11 peptide (MBP Ac1-11) for 3 days. (A) Cells were collected and adoptively transferred into B10.PL mice $\left(10 \times 10^{6}\right.$ cells per mouse). Number of mice that had clinical sign/total number of mice in each group in this representative experiment was shown as follows: anti-CD3/ CD28 (0/7) and APC/Ag (9/9). (B) Supernatants were analyzed by ELISA for IFN- $\gamma$, IL-17A, and GM-CSF. Data is representative of 3 independent experiments (mean $\pm \mathrm{SEM}) .{ }^{* *} P<0.001$ (Student's $t$ test).

IL-17-producing subset, and transferring IL-23-treated myelin-specific Th17 cells induced EAE, revealing another pathogenic mechanism in CNS autoimmunity mediated by Th17 cells (22). IL-17 transcripts and proteins were found to be present in MS lesions $(23,24)$. However, IL-17 was shown to be nonessential for EAE development $(25,26)$, indicating this Th17 signature cytokine is also not a critical element of an encephalitogenic T cell, similar to IFN- $\gamma$. IL-6 has been shown to be a critical cytokine in Th17 differentiation (27-29). Moreover, loss of IL-6 or its downstream signaling molecule, STAT3, protected mice from developing EAE $(30,31)$. Although TGF- $\beta$ plus IL-6 drove Th17 differentiation efficiently in vitro, these cells failed to transfer EAE. We and others have shown that myelin-reactive Th17 generated with IL-6 in the absence of Th1/Th2 differentiation cytokines are highly encephalitogenic (32-35). These data further confirmed that signaling components of the Th17 differentiation pathway determined the encephalitogenicity of Th17 cells but not IL-17 production itself.

Thus, the factors that are required to generate encephalitogenic $\mathrm{CD} 4^{+} \mathrm{T}$ cells are still poorly understood. It is still unclear why loss of a molecule in the Th1 differentiation pathway, such as STAT4, would negate the encephalitogenicity of Th17 cells if Th1 and Th17 cells have mutually exclusive differentiation pathways. One possibility is that there may be shared determinants of encephalitogenicity between Th1 and Th17 cells. Instead of targeting specific $\mathrm{T}$ helper-associated molecules and then investigating its impact on encephalitogenicity, we focused on what signals make $\mathrm{T}$ cells encephalitogenic, regardless of $\mathrm{T}$ helper phenotype.

\section{Results}

Contribution of APCS to the development of encephalitogenic T cells. Although the lineage-defining cytokines IFN- $\gamma$ and IL-17 are not required for EAE development $(18,19,25,26)$, it is clear that specific factors present during $\mathrm{T}$ cell differentiation promote the development of encephalitogenic $\mathrm{T}$ cells. To determine if APC-produced cytokines are critical to the development of encephalitogenic T cells, TCR Tg myelin-specific $\mathrm{CD} 4^{+} \mathrm{T}$ cells were activated with either $\mathrm{APC} / \mathrm{Ag}$ or anti-CD3/CD28 to mimic APC activation and transferred to naive recipients. Mice receiving APC/Ag-activated myelin basic protein-specific (MBP-specific) cells developed EAE, while mice that received anti-CD3/CD28-activated MBP-specific cells did not (Figure 1A). T cells activated with APC/Ag or anti-CD3/CD28 proliferated equally and expressed comparable levels of the activation marker CD44 (data not shown). This result illustrated that APCs provided a critical third signal essential for the development of encephalitogenic $\mathrm{T}$ cells.

Prior to adoptive transfer, cytokine expression of the $\mathrm{CD} 4^{+} \mathrm{T}$ cells was determined. There were only modest amounts of IFN- $\gamma$ and IL-17 detected in the supernatants, with APC/Ag-activated T cell cultures having slightly higher levels compared with anti-CD3/CD28-activated cells (Figure 1B). GM-CSF, a proinflammatory cytokine produced by encephalitogenic $\mathrm{T}$ cells (36-39), was produced in comparable amounts, irrespective of activation method (Figure 1B).

Defining the minimum cytokines that can recapitulate the signal provided by APCs to generate encephalitogenic $T$ cells. In order to identify what cytokines function as the third signal that promotes the encephalitogenicity of $\mathrm{T}$ cells, an in vitro culture system was used to recapitulate the microenvironment provided from APCs to T cells. Initially, splenocytes from naive MBP-specific TCR Tg mice were stimulated with anti-CD3/CD28 in the presence of various cytokines that had previously been shown to play a role in the differentiation of Th1 and Th17 cells - including IL-1 $\beta$, IL-6, IL-12, IL-18, IL-23, IL-27, IFN- $\gamma$, and TGF- $\beta$ - and transferred 
Table 1. Defining the cytokines required for generating pathogenic $T$ cells using anti-CD3/CD28 stimulation of myelin-specific CD4 T cells in the presence of Th1and Th17-associated differentiation cytokines

\begin{tabular}{|c|c|c|c|}
\hline Cytokine & EAE incidence & Disease severity ${ }^{A}$ & Mean max clinical score ${ }^{B}$ \\
\hline None & $0 / 27$ & NA & 0 \\
\hline IL-1 $\beta$ & $0 / 8$ & NA & 0 \\
\hline IL-6 & $9 / 20$ & $\downarrow$ & $0.9^{c}$ \\
\hline IL-12 & $25 / 32$ & $\downarrow$ & $2.2^{\mathrm{C}}$ \\
\hline IL-18 & $1 / 7$ & $\downarrow$ & $0.1^{c}$ \\
\hline IL-23 & $3 / 9$ & $\downarrow$ & $1.1^{\mathrm{c}}$ \\
\hline IL-27 & $0 / 6$ & NA & 0 \\
\hline IFN- $\gamma$ & $0 / 6$ & NA & 0 \\
\hline TGF- $\beta$ & $0 / 3$ & NA & 0 \\
\hline IL-1 $\beta+$ IL-6 & $2 / 11$ & $\downarrow$ & $0.5^{c}$ \\
\hline IL-1 $\beta+$ IL-12 & $0 / 5$ & NA & 0 \\
\hline IL-1 $\beta+$ IL-23 & $0 / 11$ & NA & 0 \\
\hline IL-1 $\beta+$ TCF- $\beta$ & $0 / 3$ & NA & 0 \\
\hline IL-6 + IL-23 & $15 / 16$ & $\leftrightarrow$ & 3.0 \\
\hline IL-6 + TCF- $\beta$ & $0 / 8$ & NA & 0 \\
\hline IL-6 + TCF- $\beta+$ IL-23 & $0 / 3$ & NA & 0 \\
\hline IL-12 + IL-23 & $7 / 8$ & $\leftrightarrow$ & 3.1 \\
\hline $\mathrm{IL}-12+\mathrm{IFN}-\gamma$ & $1 / 7$ & $\downarrow$ & $0.3^{c}$ \\
\hline IL-18 + IL-23 & $1 / 6$ & $\downarrow$ & $0.5^{c}$ \\
\hline IL-27 + IL-23 & $0 / 6$ & NA & 0 \\
\hline IFN- $\gamma+$ IL -23 & $0 / 5$ & NA & 0 \\
\hline Ag/APC & $16 / 18$ & & 3.5 \\
\hline
\end{tabular}

${ }^{A}$ NA-Not applicable; $\downarrow$ decreased disease course severity compared with antigen/APCstimulated T cells; $\leftrightarrow$ comparable disease severity to antigen/APC-stimulated T cells. ${ }^{B}$ The maximum clinical score from each mouse in each group was averaged. The mean maximum clinical scores of each group were compared with the mean maximum clinical score of the mice that received antigen/APC-activated CD4 T cells (last row). ${ }^{\mathrm{C}} P<0.05$. into WT recipients. The incidence and/or severity of EAE were low with all $\mathrm{T}$ cells cultured with individual cytokines (Table 1, Figure 2A, and Supplemental Figure 1; supplemental material available online with this article; https://doi.org/10.1172/ jci.insight.91663DS1). In contrast, differentiation with IL-6+IL-23 or IL-12+IL-23 consistently generated highly encephalitogenic cells. Figure 2A shows a representative experiment using whole splenocyte cultures, comparing IL-6, IL-23, and the combination of IL- $6+$ IL-23. To confirm that IL-6+IL-23 or IL-12+IL-23 were specifically acting on naive $\mathrm{CD}^{+} \mathrm{T}$ cells, $\mathrm{CD} 62 \mathrm{~L}^{+} \mathrm{CD} 44^{-} \mathrm{CD} 4^{+}$ $\mathrm{T}$ cells were isolated from the MBP-specific TCR $\mathrm{Tg}$ mice to eliminate potential false-positive results caused by in vivo-differentiated effector $\mathrm{T}$ cells that may be present in the splenocytes. Transfer of the myelin-specific CD62 $\mathrm{L}^{+} \mathrm{CD} 44^{-} \mathrm{CD} 4^{+} \mathrm{T}$ cells activated with anti-CD3/CD28 in the presence of IL-6, IL-12, IL-23, or combinations resulted in similar data as the total splenocytes (Figure 2B). Although both IL-6 and IL-23 have been shown to be essential for $\operatorname{EAE}(21,30)$, individually, neither of these cytokines could efficiently generate encephalitogenic T cells. In contrast, MBP-specific $\mathrm{T}$ cells that were activated with the combination of IL-6+IL-23 induced EAE similar to APC/Ag-stimulated $\mathrm{CD}^{+}{ }^{+} \mathrm{T}$ cells, suggesting IL- 6 and IL-23 synergistically promoted the encephalitogenicity in $\mathrm{T}$ cells. IL-12 is not required for EAE development $(18,19)$, and IL-12-activated MBP-specific T cells were less encephalitogenic (Table 1, Figure 2B, and

Supplemental Figure 1). Surprisingly, the combination of IL-12+IL-23 also recapitulated the third signal that generated highly encephalitogenic T cells. This result indicated a synergistic mechanism between IL-12 and IL-23 in promoting encephalitogenicity, even though these two cytokines were previously thought to regulate distinct $\mathrm{T}$ cell differentiation pathways.

IL-6 and IL-12 initiate the expression of IL-23R on naive T cells. Despite the fact that IL-23 can expand the pathogenic population in T effector cells (22), naive T cells respond poorly to IL-23 due to limited expression of the receptor for IL-23 (40). It had previously been demonstrated that IL- 6 enhanced IL-23 receptor (IL-23R) mRNA levels $(41,42)$, and thus, it is logical that naive T cells differentiated with IL- 6 would be responsive to IL-23. However, it was not clear why naive T cells differentiated with IL-12 would be responsive to IL-23. We hypothesized that both IL-6 and IL-12 induced IL-23R expression on naive T cells. To test this hypothesis, naive MBP-specific TCR Tg T cells were activated with anti-CD3/CD28 in the presence IL-23, IL-6, and/or IL-12 and were analyzed by flow cytometry. As expected, the percentage of IL-23R ${ }^{+}$cells in the IL-23-only condition was very low but increased in IL-6 and IL-12 conditions (Figure 2C). Moreover, there were substantially distinct IL-23R ${ }^{+}$populations in IL-6+IL-23 condition and IL-12+IL-23 condition. The levels of IL-23R positively correlated with the encephalitogenicity of $\mathrm{T}$ cells generated with different conditions. To confirm the flow cytometric data, the mRNA level of IL-23R was measured. Naive CD4 ${ }^{+} \mathrm{T}$ cells from WT mice were stimulated with combinations of IL-23, IL-6, and IL-12 in an APC-free system, consisting of antiCD3/CD28 without feeder cells. The level of IL-23R was significantly increased in IL-6 and IL-12 conditions compared with the IL-23 condition, which was enhanced with the addition of IL-23 (Figure 2D).

Analysis of the Th1 and Th17 cell signature cytokines found that T cells activated with IL-23 only had minimal cytokine production by both ELISA and intracellular cytokine staining (data not shown). CD4 ${ }^{+}$ $\mathrm{T}$ cells differentiated with IL-12 or IL-12+IL-23 produced high levels of IFN- $\gamma$ and low levels of IL-17 
A

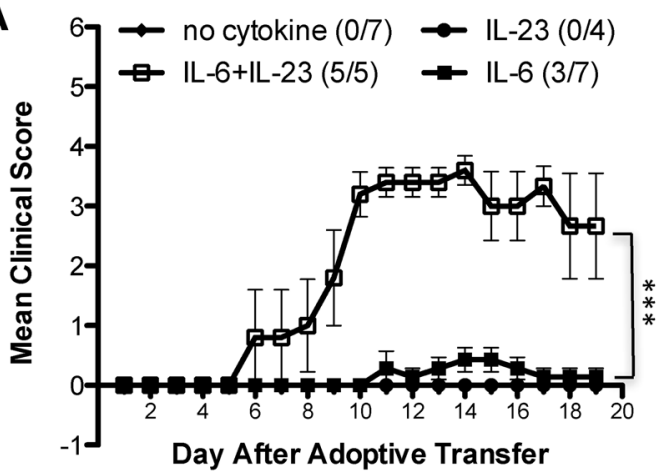

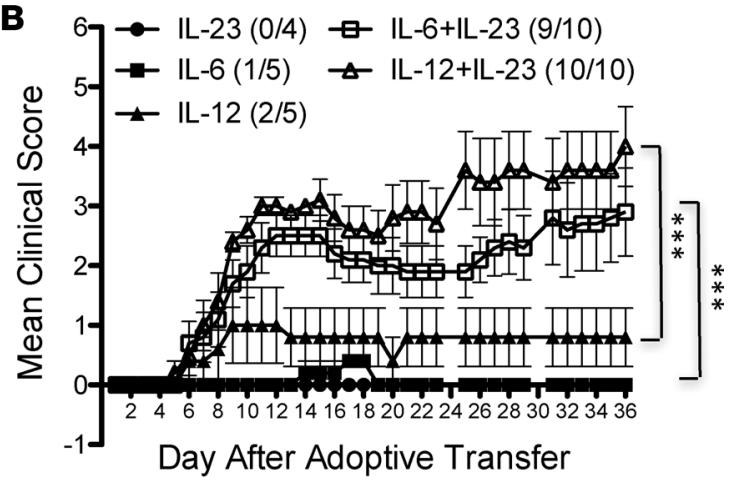

C

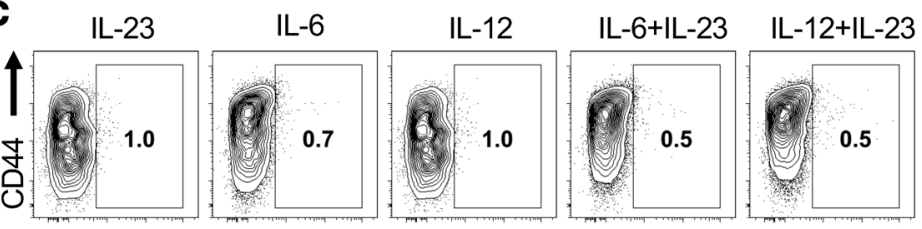
isotype
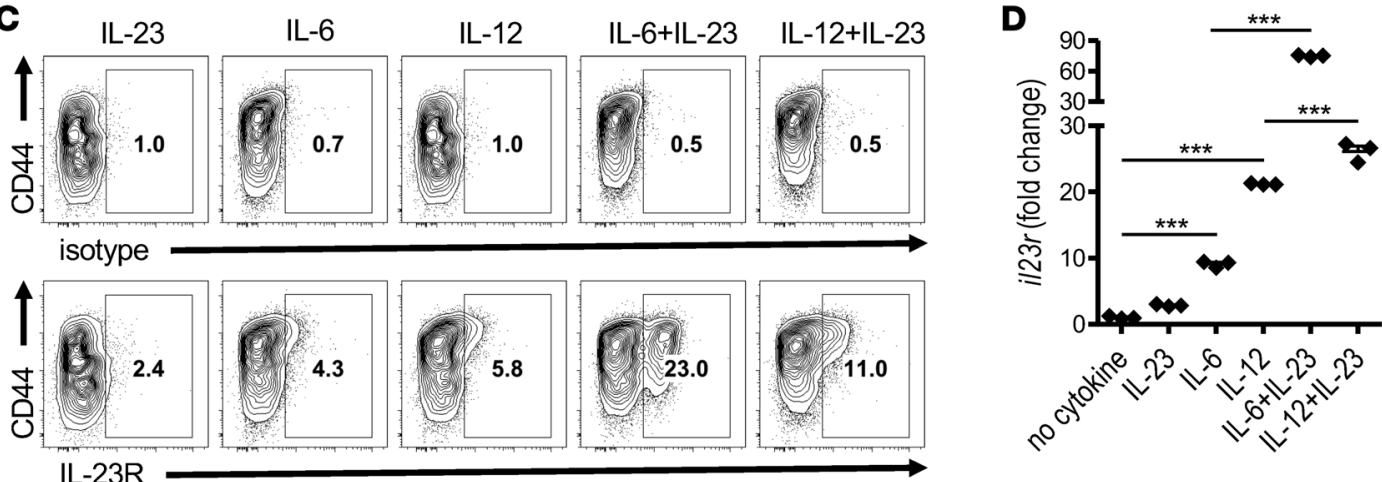

E

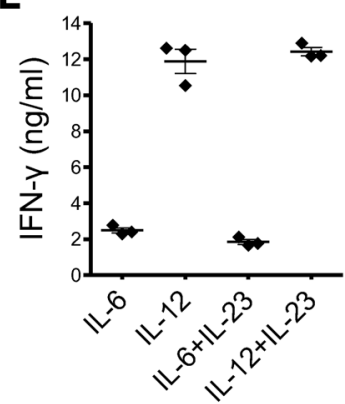

$\mathbf{F}$

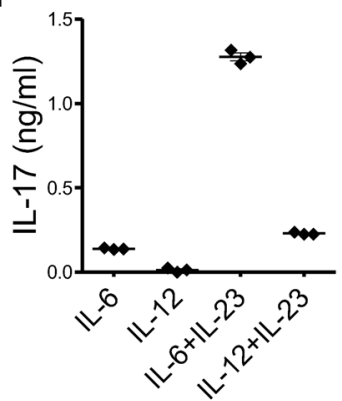

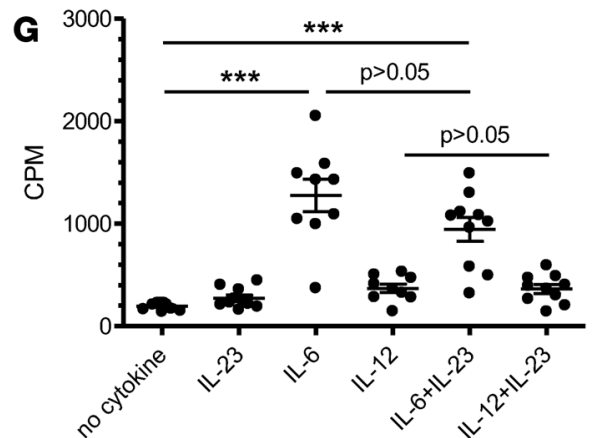

Figure 2. The combinations of IL-6+IL-23 or IL-12+IL-23 restore the encephalitogenicity to anti-CD3/CD28-activated T cells. (A) Splenocytes from V $\alpha 2.3 /$ V $\beta 8.2$ TCR Tg mice were activated in vitro with anti-CD3/CD28 with or without IL-23 and/or IL-6. At 60 hours, cells were harvested and adoptively transferred into B10.PL mice $\left(5 \times 10^{6}\right.$ cells/mouse). The number of mice with clinical signs/total number of mice in each group in this representative experiment is shown as follows: no cytokine (0/7); IL-23 (0/4); IL-6 (3/7); and IL-6+IL-23 (5/5). (B) Naive CD4+ T cells were purified from V $\alpha 2.3 / \mathrm{V} \beta 8.2 \mathrm{Tg}$ splenocytes and activated with anti-CD3/CD28 in the presence of IL-23, IL-6, and/or IL-12. At 60 hours, cells were harvested and adoptively transferred into B10.PL mice (1 $\times 10^{6}$ cells per mouse). The number of mice with clinical signs/total number of mice in each group in this representative experiment is shown as follows: IL-23 (0/4); IL-6 (1/5); IL-12 (2/5); IL-6+IL-23 (9/10); and IL-12+IL-23 (10/10). ${ }^{* *} P<0.001$ (Mann-Whitney U test). IL-23R expression (gated on CD4 ${ }^{+}$cells) was analyzed by flow cytometry (C), and supernatants were analyzed by ELISA for IFN- $\gamma(\mathbf{E})$ and IL-17A (F) (mean \pm SEM). (D) Naive CD4 ${ }^{\dagger}$ T cells were purified from B10.PL splenocytes and activated in vitro with anti-CD3/CD28 and IL-23, IL-6, IL-12, or combinations. Cells were collected at 60 hours, and II23r and Hprt mRNA were detected by real-time PCR. Fold change of gene expression was shown relative to no-cytokine condition (mean \pm SEM). (C) Proliferation was determined by ${ }^{3} \mathrm{H}$-thymidine incorporation of $\mathrm{V} \alpha 2.3 / \mathrm{V} \beta 8.2 \mathrm{TCR}$ Tg T cells using anti-CD3/CD28 stimulation with IL-6, IL-12, IL-23, or combinations. Each dot represents a replicate well. ${ }^{* *} P<0.001$ (1-way ANOVA with Bonferroni's multiple comparison test). Data is representative of $\geq 3$ independent experiments.

in the presence of IL-23 (Figure 2, E and F). Consistent with its profound effect in promoting IL-17 production that has been previously reported (43), addition of IL-23 in the presence of IL- 6 enhanced IL-17 production (Figure $2 \mathrm{~F}$ ). These data suggested that IL-23R expression was not restricted to Th17 cells and both IL-6 and IL-12 were capable of inducing IL-23R expression. Neither the number of cells producing a particular cytokine, nor the level of a particular cytokine - including GM-CSF (data not shown) - were associated with the ability of the specific T cells populations to induce EAE. T cell proliferation was not 


\section{Table 2. Genes differentially expressed in both IL-6+IL-23 and IL-12+IL-23 differentiated murine CD4 T cells}

\begin{tabular}{|c|c|c|}
\hline Gene & IL-6+IL-23A Fold change & IL-12+IL-23B Fold change \\
\hline 1122 & 22.07 & 10.25 \\
\hline Enpp2 & 11.99 & 8.35 \\
\hline Gent2 & 8.39 & 1.82 \\
\hline Lum & 6.02 & 3.93 \\
\hline Dab2 & 5.85 & 1.34 \\
\hline Tnfrsf8 & 5.65 & 1.67 \\
\hline Ermn & 4.45 & 1.92 \\
\hline Casp6 & 4.01 & 1.29 \\
\hline $\mathrm{Zc} 3 \mathrm{H} 12 \mathrm{c}$ & 3.80 & 1.45 \\
\hline Ltb4r1 & 3.67 & 1.57 \\
\hline Gatm & 3.02 & 1.91 \\
\hline Ccr2 & 2.84 & 2.05 \\
\hline Crispld2 & 2.56 & 1.33 \\
\hline Cd163l1 & 2.48 & 2.15 \\
\hline Avpi1 & 2.28 & 1.41 \\
\hline Gnpda2 & 2.25 & 1.20 \\
\hline Penk & 0.56 & 0.66 \\
\hline
\end{tabular}

${ }^{A}$ Fold change was determined by subtracting the average gene expression level observed in IL-6 differentiated $C D 4^{+} T$ cells from the IL-6+IL-23 differentiated CD4 T cells. The threshold for selection was a fold change $>2$ and $P<0.01$. ${ }^{B}$ Fold change was determined by subtracting the average gene expression level observed in IL-12 differentiated CD4 ${ }^{+} T$ cells from the IL-12+IL-23 differentiated CD4 T cells. The threshold for selection was $P<0.02$. influenced by the addition of IL-23 (Figure 2G), suggesting that enhancement of IL-23R expression was not due specifically to the expansion of the IL-23R ${ }^{+}$ $\mathrm{T}$ cells, but to an increase in the number of IL-23R $\mathrm{R}^{+} \mathrm{T}$ cells.

Analysis of transcription factors, activation markers, and chemokine receptors by real-time PCR did not find any association of these molecules with encephalitogenicity (Supplemental Figure 2). Therefore, a microarray analysis of purified myelin-specific $\mathrm{CD}^{+} \mathrm{T}$ cells differentiated with anti-CD3/CD28 in the presence of IL- 6 versus IL- $6+$ IL-23 or IL-12 versus IL-12+IL-23 was performed to determine global differences in gene expression in the encephalitogenic T cells (Supplemental Tables 1-4). There were 16 genes that were upregulated and 1 gene downregulated common to both populations of encephalitogenic $\mathrm{CD} 4^{+} \mathrm{T}$ cells (Table 2). Il22, Enpp2, Lum, and $C c r 2$ were the most significantly differentially expressed genes using a $P<$ 0.01 and fold change $>2$ as the threshold for analysis. Pathway analysis of the common differentially expressed genes in Table 2 did not identify any specific pathway altered by the addition of IL-23, suggesting that IL-23 signaling is likely altering specific molecules in one or more pathways that are required for encephalitogenicity.

IL-6 and IL-12 provided by APC is critical for promoting the encephalitogenicity in naive T cells. To verify that IL-23R signaling was secondary to the upregulation of IL-23R by IL-6 and IL-12 for generation of encephalitogenic T cells, myelin-specific $\mathrm{T}$ cells were activated with anti-CD3/CD28 in the presence or absence of IL- 6 or IL-12 only for 48 hours, and IL-23 was added in the cultures only during the 18 hours immediately prior to transfer of the cells into mice. EAE was restored in the T cells differentiated with IL-6 followed by IL-23 and IL-12 followed by IL-23, but not with the cells that only received IL-23 (Figure 3A), indicating that IL-23R signaling is not required prior to the upregulation of IL-23R by IL-6 or IL-12. The addition of IL-23 to the cultures had no effect on the proliferation of the cells (Figure 3B).

To confirm whether IL- $6+$ IL-23 or IL-12+IL-23 were the minimal essential factors provided by APCs as the critical third signal, as well as to address the contributions of IL- 6 and IL-12 to the encephalitogenicity of T cells, we examined the encephalitogenicity of APC/Ag-activated T cells while blocking IL- 6 or IL-12 in culture. Splenocytes from MBP-specific TCR Tg mice were activated with MBP Ac1-11 peptide, and IL-6 and/or IL-12 neutralizing Ab were added during the in vitro differentiation. The cells were then adoptively transferred into B10.PL recipients. Blocking IL-6, IL-12, or both IL-6 and IL-12 reduced the incidence and severity of disease compared with APC/Ag (Figure 3C). IL-6 was efficiently neutralized (Figure 3D). IL-12 was not detectable in all conditions (data not shown), but there was a 2-fold reduction of IFN- $\gamma$ in the presence of anti-IL-12 (Figure 3D), suggesting IL-12 was at least partially blocked. The levels of IL-17 were very low in all conditions, and anti-IL-6 resulted in variable decreases in IL-17 levels ranging from $0 \%-60 \%$ in multiple experiments (Figure 3D and data not shown). The levels of GM-CSF were very similar in all culture conditions, irrespective of the encephalitogenicity (Figure 3D), indicating that the level of GM-CSF was not a specific marker for encephalitogenicity.

IL-23 stimulation results in phosphorylation of STAT4. It has been shown that STAT3 is activated upon IL-23 stimulation and recognized as essential for IL-17 production (41). IL-23 has been shown to activate STAT4 but to a lesser extent than IL-12 $(44,45)$. IL-23-induced IFN- $\gamma$ production in Th17 precursor cells is STAT4 dependent (46). Furthermore, STAT4 has been shown to be partially required for the development of IL-23primed IL-17-producing cells (47), but the functional role of STAT4 upon IL-23 stimulation is not known. It had previously been shown that IL-12 is not an essential cytokine for the differentiation of encephalitogenic $\mathrm{T}$ cells $(18,19)$, yet STAT4 - the dominant transcriptional regulator of the IL-12 receptor signaling pathway is essential (20), so we investigated whether the essential role for STAT4 lies in the IL-23R signaling pathway. WT lymphocytes were activated with anti-CD3/CD28 in the presence of IL-23, IL-6, and IL-12 or combinations (Figure 4). The cells were then washed and rested in fresh medium for 4 hours, allowing the residual signal transduction that was induced by culture condition to partially return to basal levels. Rested cells were 

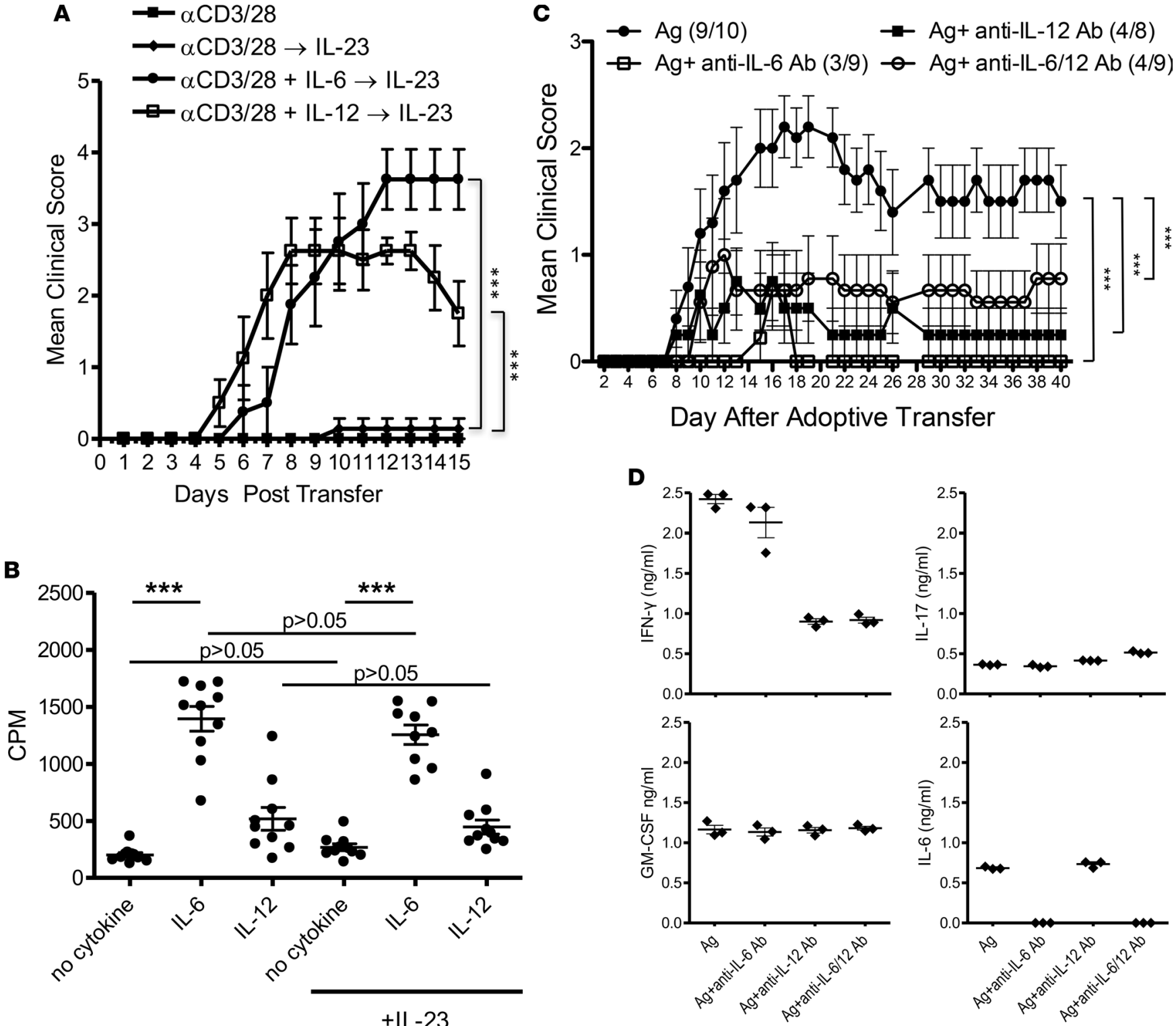

Figure 3. Encephalitogenicity is dependent on IL-6 or IL-12 provided by APCs. (A) Lymphocytes from naive V $\alpha 2.3 / \mathrm{V} \beta 8.2$ TCR Tg mice depleted of APCs were activated with anti-CD3/CD28 with IL-6, IL-12, or no cytokines for 48 hours. IL-23 was added for the last 18 hours of culture prior to transfer into B10.PI mice (5 $\times 10^{6}$ cells/mouse). Anti-CD3/CD28 only ( $n=6$ mice), anti-CD3/28 with IL-23 during last 18 hours $(n=7)$, anti-CD3/28+IL-6 with IL-23 during last 18 hours ( $n=$ 8), and anti-CD3/28+IL-12 with IL-23 during last 18 hours $(n=8)$. ${ }^{* *} P<0.001$ (Mann-Whitney $U$ test). (B) Proliferation of these cells was determined by adding ${ }^{3} \mathrm{H}$-thymidine during the last 18 hours of parallel cultures. ${ }^{* *} P<0.001,1$-way ANOVA with Bonferroni's multiple comparison test. (C) Splenocytes from naive V $\alpha 2.3 / \mathrm{V} \beta 8$ 8.2 TCR Tg mice were activated with MBP Ac1-11 peptide for 3 days in the absence/presence of neutralizing Ab to IL-6 and/or IL-12. Cells were collected, and $10 \times 10^{6}$ cells per mouse were adoptively transferred into naive B10.PL mice. The number of mice with clinical signs/total number of mice in each group in this representative experiment is shown as follows: Ag (9/10); Ag+anti-IL-6 (3/9); Ag+anti-IL-12 (4/8); and Ag+anti-IL-6/12 (4/9). ${ }^{* * *} P<0.001$ (Mann-Whitney $U$ test). (D) Supernatants were analyzed by ELISA for IFN- $\gamma$, IL-17, IL-6, and GM-CSF. Data is representative of 3 experiments (mean \pm SEM).

stimulated with PBS or IL-23. The expression of p-STAT3 and p-STAT4 was analyzed in CD4 T cells by flow cytometry (Figure 4, A and B), and the degree of phosphorylation of STAT3 and STAT4 in response to IL-23R signaling in whole cell lysates was demonstrated by Western blot (Figure 4, C-E). Flow cytometric analysis showed that IL-23 enhanced p-STAT4 in both IL-6- (Figure 4A) and IL-12-differentiated (Figure 4B) CD4 T cells. This was confirmed by Western blot. ImageJ software was used to quantify band intensities. The ratio of phosphorylation was determined by comparing phosphorylated STAT protein to total STAT protein, and the no-cytokine condition in the PBS-treated group was used as reference for normalization. The comparisons between IL- 6 and IL- $6+$ IL-23 conditions were shown in Figure 4D, and comparisons between IL-12 and IL-12+IL-23 conditions were shown in Figure 4E. In the PBS-treated group, high basal levels of 
p-STAT3 were observed in the IL-6+IL-23 condition, which was likely due to the actions of IL-6 and IL-23 in the initial culture, since both are known to signal through p-STAT3. Secondary IL-23 stimulation resulted in the highest elevation of STAT3 phosphorylation in IL-6+IL-23 condition (Figure 4D, left). IL-23-induced STAT3 phosphorylation was also seen in IL-12 and IL-12+IL-23 conditions, and p-STAT3 was higher in the presence of IL-12+IL-23 than IL-12 only (Figure 4E). Phosphorylation of STAT4 was maintained at low levels in the PBS-treated group, except for the IL-12 and IL-12+IL-23 conditions, which had residual STAT4 activation (Figure 4E, right). Compared with the PBS group, secondary IL-23 stimulation enhanced STAT4 phosphorylation in the IL-6 condition and dramatically enhanced STAT4 phosphorylation in the IL-6+IL-23 condition (Figure 4D). Furthermore, the level of p-STAT4 in IL-6+IL-23 was substantially higher than in the IL-6 condition (Figure 4D). These data suggested that IL-23 stimulation triggered phosphorylation of STAT3 and STAT4 in IL-23R-expressing T cells that were activated with IL-6+IL-23 or IL-12+IL-23.

Suppressing STAT4 expression reduces encephalitogenicity of $T$ cells differentiated without IL-12. Although STAT4 plays a major role in IL-12 signaling (48), STAT4-deficient mice are resistant to EAE (20), but IL-12-deficient mice are not $(19,49)$. Why deletion of 2 molecules in the same pathway resulted in opposite outcomes was unknown. The participation of STAT4 in signaling by IL-23 was one possibility. To demonstrate whether STAT4 was critical for IL-23R-associated encephalitogenicity, we compared the capability of inducing EAE between STAT4 knock-down and control T cells. MBP-specific TCR lymphocytes were transfected with STAT4 siRNA (si-STAT4) or non-sense siRNA (si-NS) overnight (Figure 5A). The transfected cells were then activated with MBP Ac1-11 peptide plus APCs in the absence of any exogenous cytokine or activation with anti-CD3/CD28 plus IL-6+IL-23. Both of these conditions generated encephalitogenic T cells in the absence of exogenous IL-12. The activated cells were adoptively transferred into B10.PL recipients, and disease course was monitored. Suppressing STAT4 expression by siRNA in T cells significantly ameliorated the disease severity caused by APC/Ag-activated cells (Supplemental Figure 3). This was not surprising, given that a previous study had found that adoptive transfer of STAT4-deficient CD4 T cells into T cell-deficient mice failed to induce EAE (20). The more critical question was whether STAT4 played a critical role in EAE mediated by T cells differentiated with IL-6+IL-23, given that this pathway was thought to be mediated by STAT3 and independent of STAT4. Similarly, mice receiving STAT4-knockdown T cells that were activated with anti-CD3/CD28 plus IL-6+IL-23 developed significantly milder EAE when compared with si-NS control (Figure 5B). An independent siRNA was used to confirm that a reduction in STAT4 levels reduces the encephalitogenicity of IL-6+IL-12-differentiated myelin-specific T cells (Supplemental Table 5). Knocking-down STAT4 resulted in a modest but significant reduction in proinflammatory cytokines (Figure 5C), but antiinflammatory cytokines (IL-10 and TGF- $\beta$ ) were below the level of detection (data not shown). There was no evidence that knockdown of STAT4 affected the activation of the CD4 T cells or skewed the differentiation toward Tregs (Supplemental Figure 4). Although a better approach may have been to use of STAT4 ${ }^{-/-} \mathrm{T}$ cells, this would require an extensive backcrossing of the STAT4 ${ }^{-/-}$mice with the B10.PL MBP-specific TCR Tg mice.

IL-23R transduces signal via p-STAT3/p-STAT4 heterodimers. Both STAT3 and STAT4 are critical for $\mathrm{T}$ cell encephalitogenicity $(20,31)$, but they were previously shown to be involved in distinct Th differentiation pathways. It remains unclear why loss of either STAT3 or STAT4 impairs both Th1- and Th17-mediated pathogenic mechanisms in EAE. One possibility is that STAT3 and STAT4 promote the encephalitogenicity of $\mathrm{T}$ cells in a synergistic fashion. Thus, deletion of either molecule results in a complete loss of a critical signal for inducing encephalitogenicity. To examine this possibility, co-IP assays were performed to identify if there is a direct interaction between p-STAT3 and p-STAT4 under IL-23R signaling. Purified $\mathrm{CD} 4^{+} \mathrm{T}$ cells from naive WT mice were activated with anti-CD3/CD28 in the presence of IL-6+IL-23 or IL-12+IL-23 in an APC-free system. After 3 days in culture, the cells were washed and rested, allowing the residual signaling cascade to partially return to basal levels. $\mathrm{CD} 4^{+} \mathrm{T}$ cells from naive WT mice served as negative controls. The 3 groups of T cells were stimulated with additional IL-23 for 30 minutes, and nuclear protein were extracted. Magnetic beads and Ab for STAT3 were used to pull down STAT3 protein complexes. We detected p-STAT4 in the inputs of nuclear extracts from IL-6+IL-23 and IL-12+IL-23 conditions but not in the control cells (Figure 5D). This confirmed our previous observation that IL-23 induced STAT4 phosphorylation in IL-23R-expressing T cells (Figure 4). Most importantly, p-STAT4 were detected in the STAT3 pull-down of T cells cultured with IL-6+IL-23 and IL-12+IL-23, but they were not detected with a control IgG. The level of p-STAT4 in the IL-12+IL-23-differentiated CD4 T cells was higher in the input and STAT3 pull-down samples, as demonstrated by its detection at 60 
A
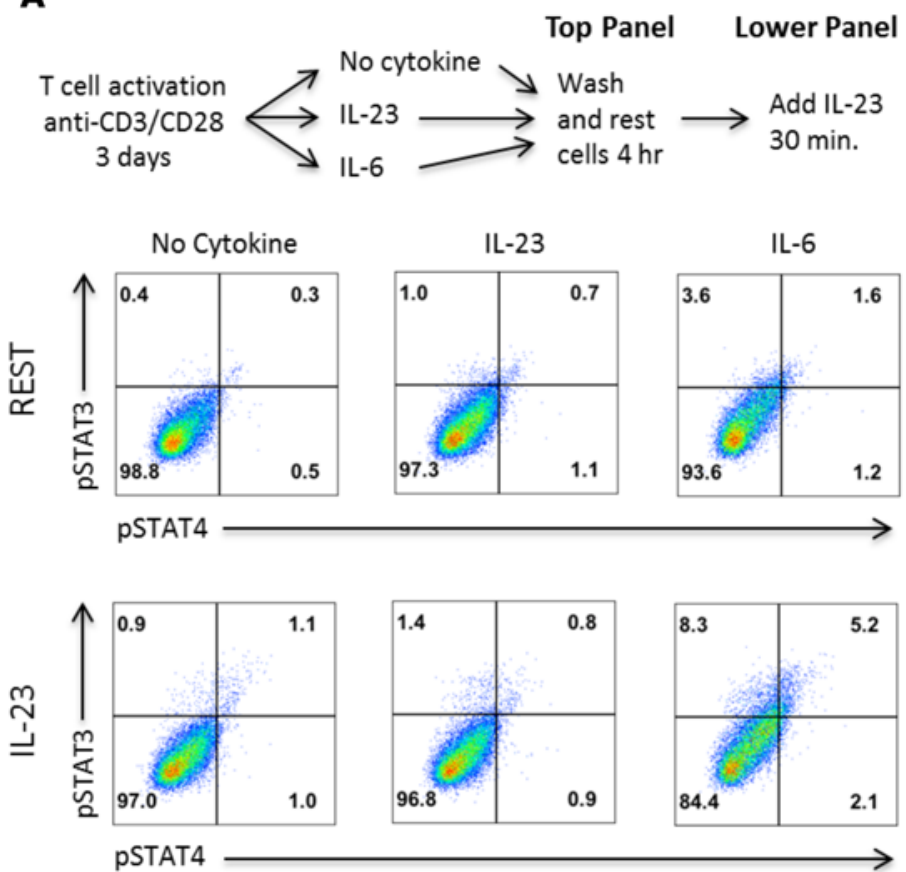

B
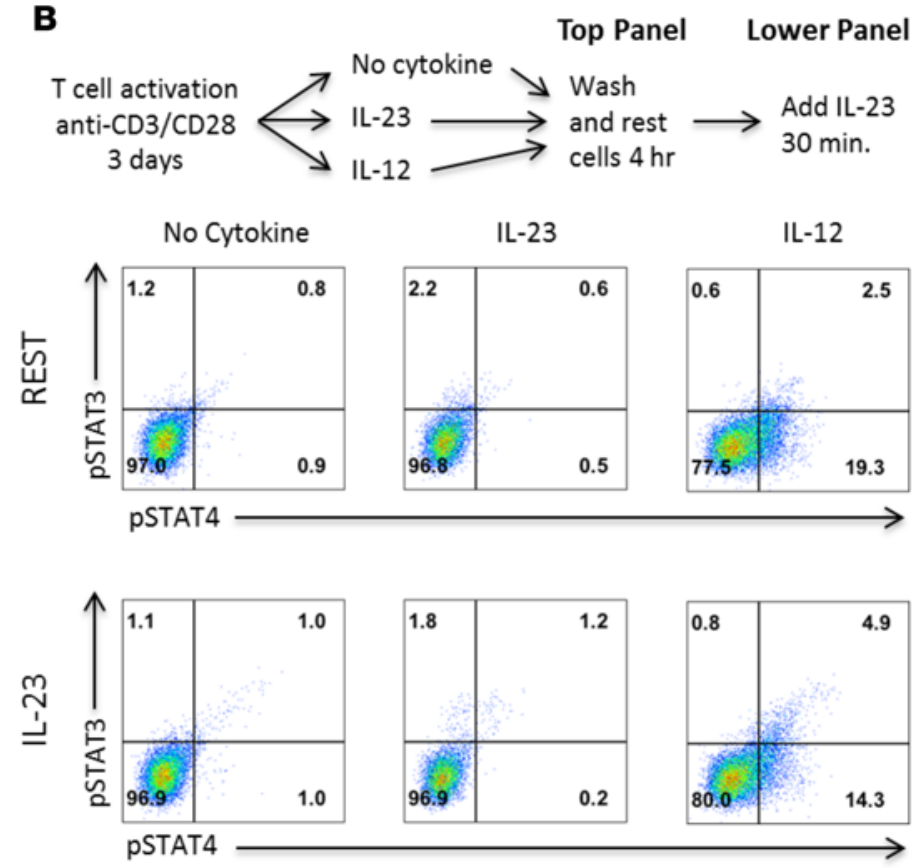

C

PBS

IL-23

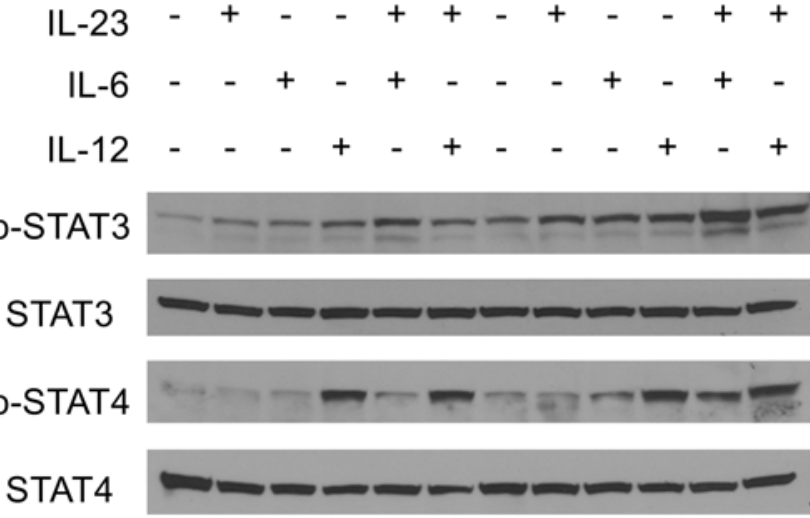

$\beta$-actin

D

PBS - $2^{\circ}$ Stimulation

IL-23 - $2^{\circ}$ Stimulation

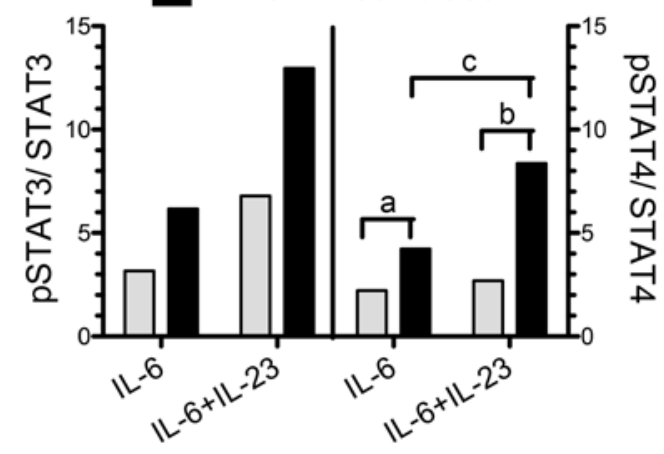

$1^{\circ}$ Stimulation

E

PBS - $2^{\circ}$ Stimulation

IL-23 - $2^{\circ}$ Stimulation

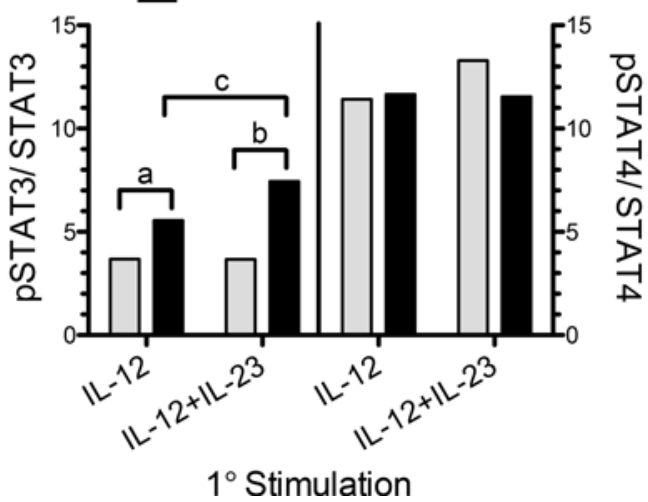

Figure 4. IL-23R signaling activates both STAT3 and STAT4. Splenocytes from naive B10.PL mice were activated in vitro with anti-CD3/CD28 in the absence/presence of IL-23, IL-6, IL-12, or combinations for 66 hours. The cells were washed and rested in fresh medium for 4 hours and then were stimulated with either IL-23 or PBS for 30 minutes. (A and B) Cells were analyzed by flow cytometry. Gated CD4 T cells were analyzed for p-STAT3 and p-STAT4. (C) Whole cell lysate was extracted, and p-STAT3, STAT3, p-STAT4, STAT4, and $\beta$-actin levels were determined by Western blotting. ( $\mathbf{D}$ and $\mathbf{E}$ ) The band intensity of STAT3, STAT4, p-STAT3, and p-STAT4 were quantified by NIH Image) software, and no cytokine condition in the PBS group was used as reference for normalization. The ratio of phosphorylation was determined by comparing p-STAT3 to STAT3 and p-STAT4 to STAT4. The data is representative of 4 independent experiments. The $a, b$, and $c$ in $\mathbf{D}$ and $\mathbf{E}$ illustrate qualitative changes in p-STAT4 (D) and p-STAT3 (E) in response to IL-23. 
A
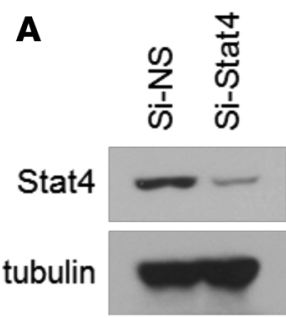

B Anti-CD3/CD28 plus IL-6+IL-23

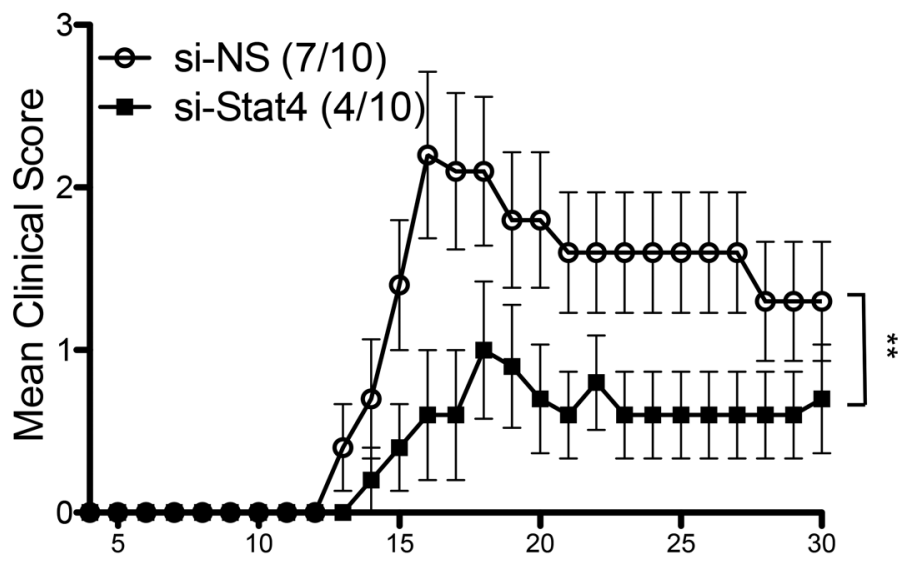

Day Post Adoptive Transfer

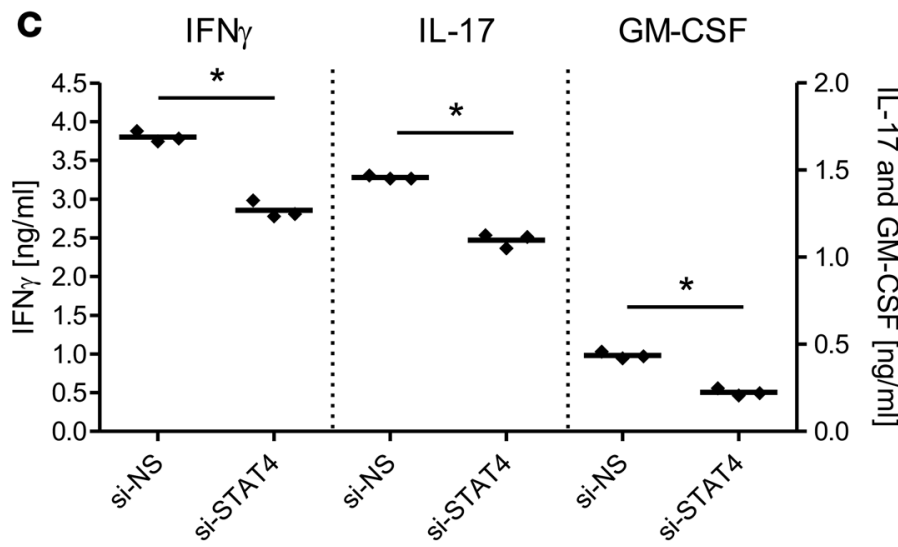

D
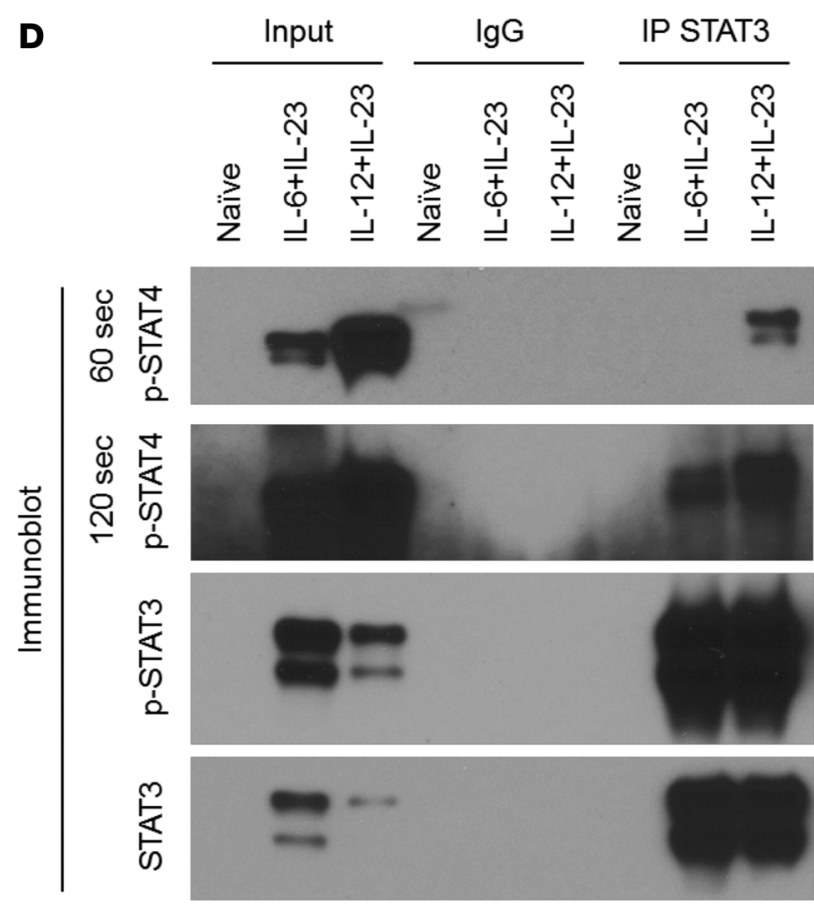

E

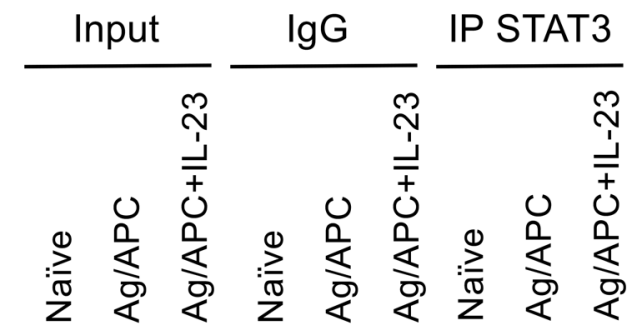

$\underline{\underline{0}}$
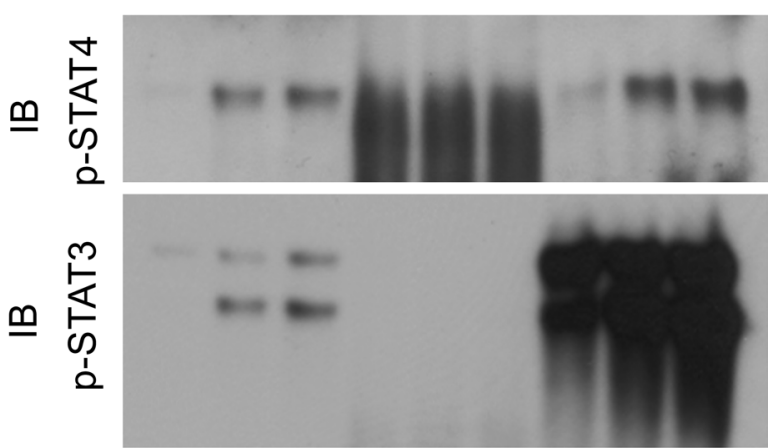

Figure 5. IL-23 induces the formation of p-STAT3/p-STAT4 heterodimers. Splenocytes from naive V $\alpha 2.3 / \mathrm{V} \beta 8.2$ TCR Tg mice were transfected with either si-NS or si-Stat4 for 18 hours. (A) Knockdown of STAT4 was determined by Western blot of si-NS and si-STAT4 transfected cells using tubulin as the control protein. (B) The si-STAT4 or si-NS transfected cells were activated with anti-CD3/CD28 plus IL-6+IL-23 for 60 hours and then adoptively transferred into naive B10.PL mice $\left(5 \times 10^{6}\right.$ cells per mouse). The number of mice with clinical signs/total number of mice in each group is shown as follows: si-NS (7/10) and si-STAT4 (4/10). (C) Supernatants from anti-CD3/CD28 plus IL-6+IL-23 culture condition were analyzed by ELISA for IFN- $\gamma$, IL-17, and GM-CSF. ${ }^{*} P<0.05$ (Mann-Whitney $U$ test). (D) Purified CD4+ $T$ cells from naive B10.PL mice were activated in vitro with anti-CD3/CD28 in the presence of the combinations of IL-6+IL-23 or IL-12+IL-23 for 64 hours. CD4+ $T$ cells from naive mice served as a negative control. The 3 groups of cells were washed and rested in fresh medium for 4 hours, and then IL-23 or PBS were added as secondary stimulations for 30 minutes. Nuclear protein was extracted for use in a co-IP assay performed with Ab for total STAT3 or IgG control. Precipitated protein complexes and inputs were electrophoresed with SDS-PAGE. p-STAT4, p-STAT3, and total STAT3 were analyzed sequentially by Western blot, and images shown are from the same blot. (E) Splenocytes from naive V $\alpha 2.3 / \mathrm{V} \beta 8.2 \mathrm{TCR}$ Tg mice were activated with MBP Ac1-11 with and without IL-23 for 64 hours, and then CD4+ T cells were purified. Nonactivated T cells were the negative control. Nuclear protein was extracted for use in a co-IP assay performed with Ab for total STAT3 and an isotype control. Precipitated protein complexes and inputs were electrophoresed with SDS-PAGE. p-STAT4 (top) and p-STAT3 (bottom) were detected by Western blot. 
seconds, while p-STAT4 was not detectable until 120 seconds in the IL-6+IL-23 STAT3 pull-down, which is logical given that IL-12 signals via p-STAT4 (Figure 5D). The same blot was probed with anti-p-STAT3 and anti-STAT3 to confirm the level of STAT3 pull-down in the samples. p-STAT3 and STAT3 were higher in the IL-6+IL-23-differentiated cell input, which is logical since both IL-6 and IL-23 are known to signal via p-STAT3. The amount of p-STAT3 and STAT3 were similar in the STAT3 pull-down in both IL-6+IL-23- and IL-12+IL-23-differentiated cells, but p-STAT3 and STAT3 were absent from the naive CD4 T cells treated with only IL-23. These data demonstrated the presence of STAT3/STAT4 heterodimers translocated into the nuclei of both IL-6+IL-23- and IL-12+IL23-differentiated cells.

To confirm that the STAT3/STAT4 heterodimers form in Ag/APC-differentiated T cells, myelin-specific splenocytes from naive mice were not activated (negative control) or were activated with MBP Ac111 in the presence or absence of exogenous IL-23. After 3 days in culture, CD4 T cells were purified and then stimulated with additional IL-23 for 30 minutes. Nuclear extracts were prepared, and Ab for STAT3 were used to pull down STAT3 protein complexes. The levels of p-STAT4 (Figure 5E, top) and p-STAT3 (Figure 5E, bottom) were determined in the inputs of the nuclear extracts, as well as in pull-down proteins from immunoprecipitation with an irrelevant IgG and STAT3 antibody. Both the input and pull down from Ag/APC-activated cells were positive for p-STAT4, while the control IgG was negative. Addition of exogenous IL-23 had only a modest increase on STAT3/STAT4 heterodimers, suggesting that the APCs provided sufficient signals to induce these heterodimers. Since loss of either STAT3 or STAT4 inhibits the differentiation of encephalitogenic T cells, these data support the hypothesis that p-STAT3/p-STAT4 heterodimers may be a key transcriptional regulator mediated by IL-23R signaling that induces genes essential for encephalitogenicity, but this will require extensive investigation to be confirmed.

IL-23R is induced by IL-6 and IL-12 on naive human CD4+ $T$ cells. To determine if IL- 6 and IL-12 both induce IL-23R expression on naive human $\mathrm{CD} 4^{+} \mathrm{T}$ cells, naive $\mathrm{T}$ cells were isolated from peripheral blood mononuclear cells (PBMC) and stimulated with anti-CD3/CD28-coated beads in the presence of IL-6, IL-12, IL-23, or a combination of IL-6+IL-23 or IL-12+IL-23. After 48 hours of stimulation, the mRNA levels of $I L 23 R$ were determined. Both IL-6 and IL-12 induced IL23R expression (Figure 6A). IL-23 enhanced the expression of IL23R in the presence of IL-6. In contrast to murine CD4+ T cells, IL-12 induced very high expression of $I L 23 R$ on human $C D 4^{+} \mathrm{T}$ cells that was not enhanced by IL-23. To further characterize the phenotype of the human $\mathrm{CD}^{+} \mathrm{T}$ cells differentiated with the different conditions, gene expression was determined for cytokines, transcription factors, activation markers, and chemokine receptors (Figure $6 \mathrm{~B}$ ). Interestingly, only $C C R 7$ and $C D 27$ gene expression pattern was associated (in an inverse manner) with the expression of IL23R.

To evaluate the sensitivity of IL-6+IL-23- and IL-12+IL-23-differentiated human CD4+ T cells to IL-23, activated human T cells were rested and restimulated with PBS or IL-23. As seen in Figure 6C, IL-23 enhanced p-STAT3 levels and the percentage of p-STAT3/p-STAT4 double-positive T cells in both conditions. Consistent with the IL23R mRNA levels, the IL-12+IL-23-differentiated T cells were more sensitive to IL-23 than the IL-6+IL-23-differentiated T cells, consistent with the higher expression of $I L 23 R$ induced with IL-12 in human CD4 ${ }^{+} \mathrm{T}$ cells.

To determine whether the IL-23R signaling may result in STAT3/STAT4 heterodimers as observed in murine $\mathrm{T}$ cells, naive human $\mathrm{CD}^{+} \mathrm{T}$ cells were differentiated with IL-6 or IL-12 initially, followed by a secondary stimulation with IL-23. STAT3/STAT4 heterodimers were detected in the both IL-6 and IL-12 differentiated CD4 ${ }^{+} \mathrm{T}$ cells, but they were not in naive $\mathrm{CD} 4^{+} \mathrm{T}$ cells or when a control $\mathrm{IgG}$ was used in the co-IP (Figure 6D). Together, these data indicate that both IL-6 and IL-12 can induce IL-23R expression on naive human $\mathrm{CD}^{+} \mathrm{T}$ cells and that IL-12 may play a larger role in IL-23R expression in human cells than IL-6. In addition, the IL-23R signaling induces both p-STAT3 and p-STAT4, which can form heterodimers, consistent with the observation that these heterodimers are present in encephalitogenic murine $\mathrm{CD}^{+} \mathrm{T}$ cells.

Memory $C D 4^{+} T$ cells from $M S$ patients are hyperresponsive to $I L-23$. Due to the essential role of IL-23 in determining the encephalitogenicity of $\mathrm{T}$ cells, we further investigated whether memory T cells from MS patients respond to IL-23 differently compared with those from healthy individuals. To restimulate memory $\mathrm{CD}^{+} \mathrm{T}$ cells, PBMCs from healthy individuals and MS patients were activated with anti-CD3 in the absence of exogenous cytokines for 48 hours. Omitting anti-CD28 from the activation promotes the activation of memory/effector $\mathrm{T}$ cells, while minimizing the activation of naive $\mathrm{T}$ cells. The cells were then washed and rested in fresh medium and then stimulated with PBS or IL-23. The levels of p-STAT3 and 
A

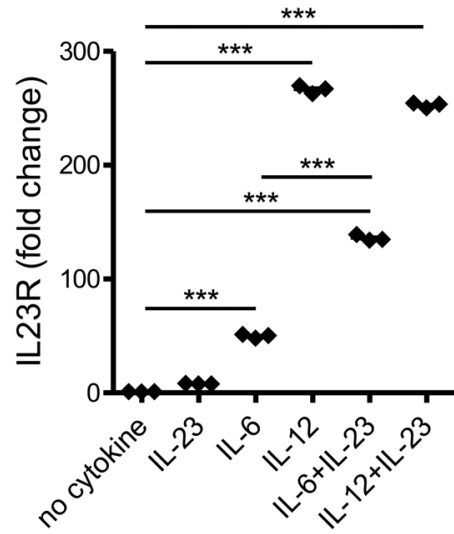

C
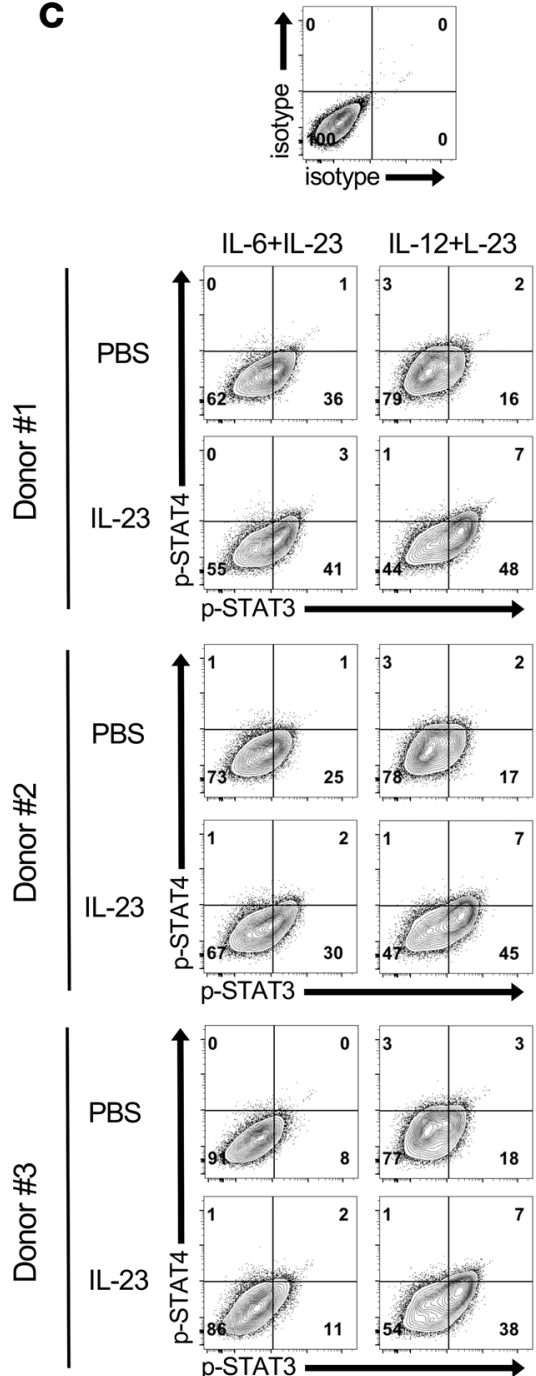

B
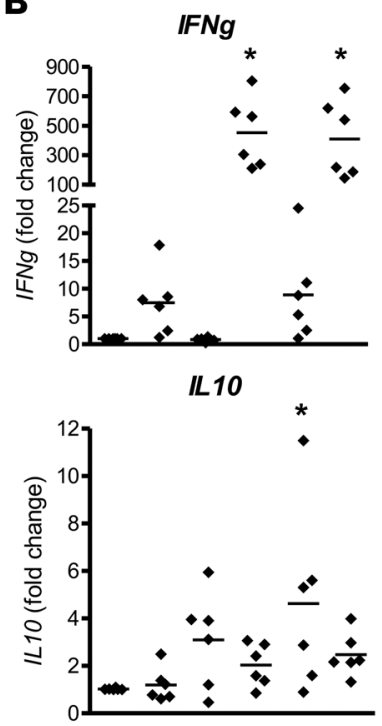

IL2RA

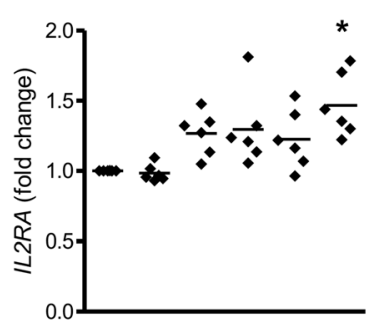

CD40L

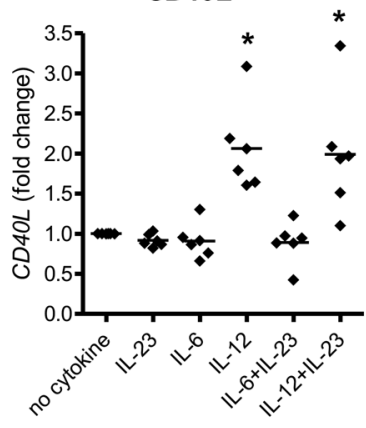

D

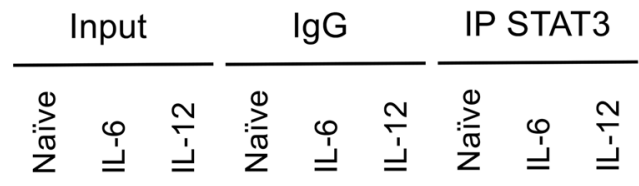

IL17A
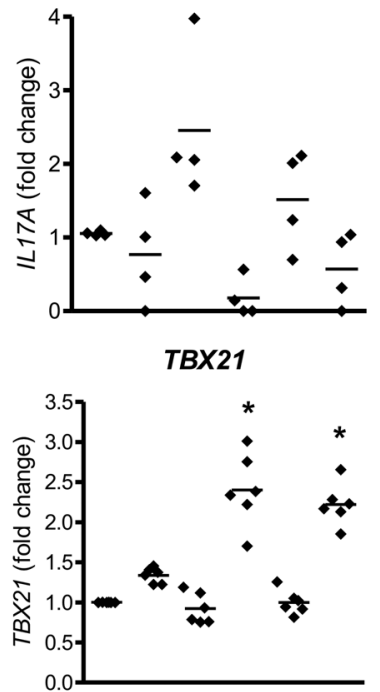

IL7R

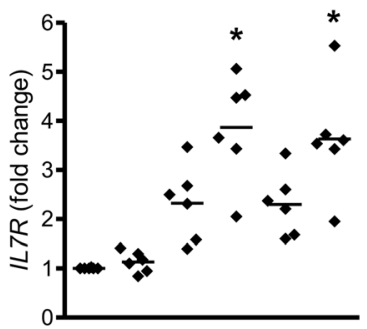

CCR7

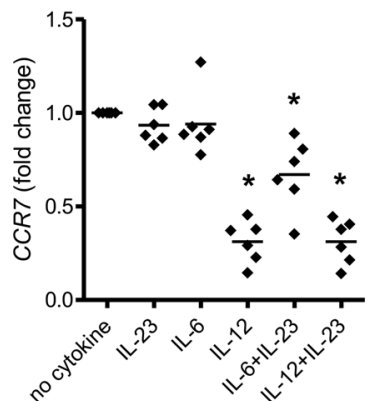

CSF2
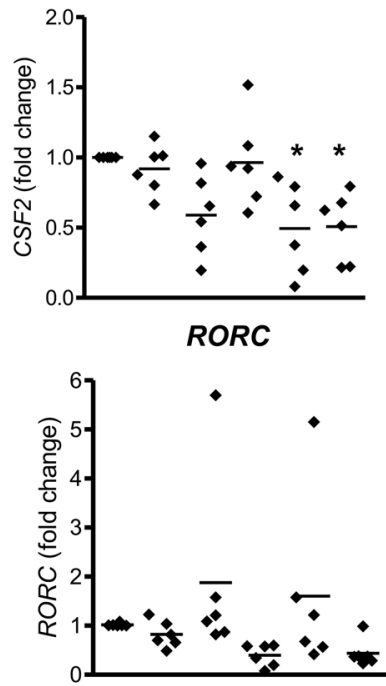

CXCR3

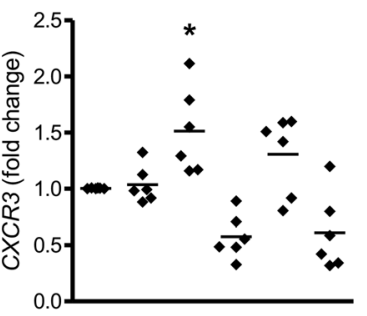

CD27
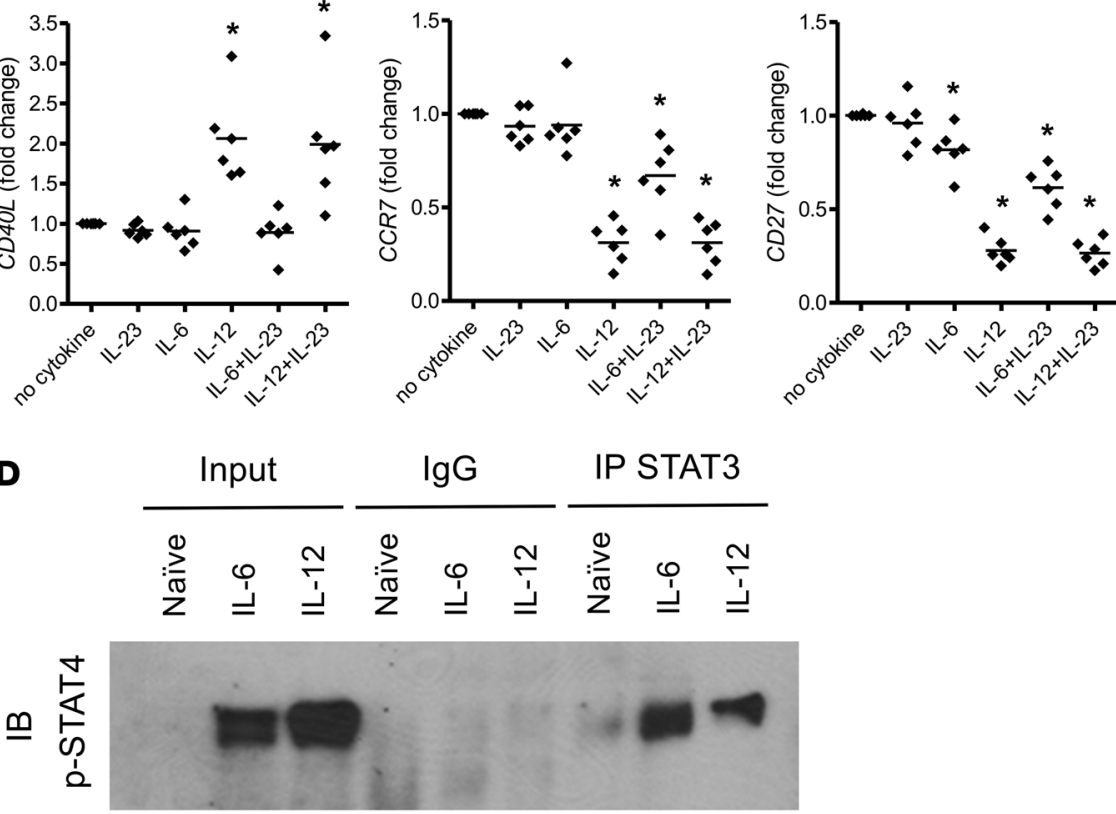

Figure 6. IL-23R signals through p-STAT3/p-STAT4 heterodimers on human CD4 ${ }^{+}$T cells differentiated IL-6 or IL-12. Naive human CD4 ${ }^{+}$T cells were purified from PBMCs of healthy donors and activated in vitro with anti-CD3/CD28-coated beads in the presence of IL-23, IL-6, IL-12, or combinations. (A) Cells were collected at 48 hours, and IL-23R and HPRT mRNA were detected by real-time PCR. Fold change of gene expression was shown relative to no cytokine condition (mean $\pm \mathrm{SEM}$ ). ${ }^{* * *} P<0.001$ (1-way ANOVA with Bonferroni's multiple comparison test). (B) Additional genes were analyzed by real-time PCR ( $n=6$ healthy donors). ${ }^{*} P<0.05$ (1-way ANOVA with Bonferroni's multiple comparison test). (C) At 72 hours, the activated T cells were washed and rested in fresh medium for 4 hours, and then IL-23 or PBS were added as secondary stimulations. After 20 minutes, phosphorylation of STAT3 and STAT4 in CD4 ${ }^{+}$T cells was analyzed by flow cytometry. (D) After 30 minutes, differentiated cells were collected, and undifferentiated naive CD4+ ${ }^{+}$cells from the same donor served as a negative control. Nuclear protein was extracted for use in a co-IP assay performed with Ab for total STAT3 or nonspecific mouse IgG. Precipitated protein complexes and inputs were electrophoresed with SDS-PACE. p-STAT4 was detected by Western blot. Data is representative of at least 2 independent experiments. 
A
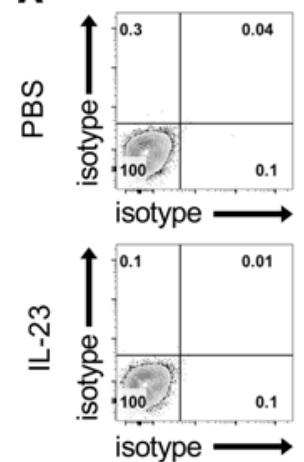

$\mathrm{HI}-1$

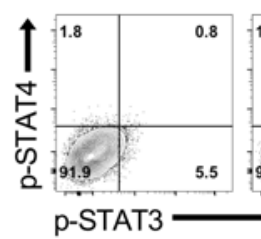

HI-2

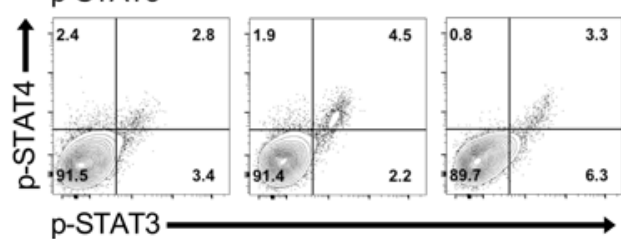

HI-3

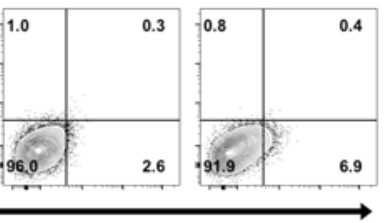

B

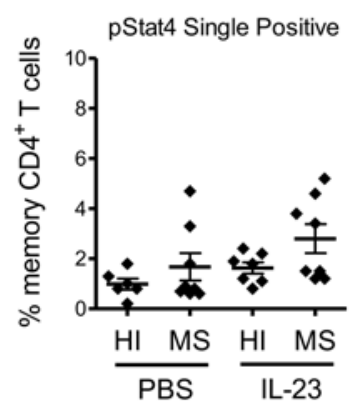

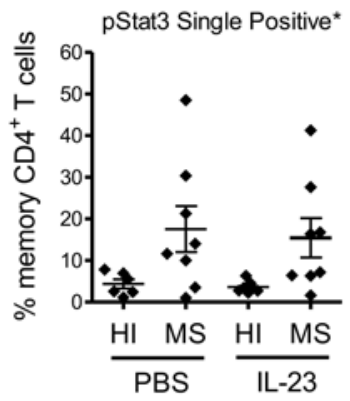

pStat3 Single Positive*

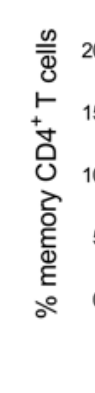

pStat3/pStat4 Positive**

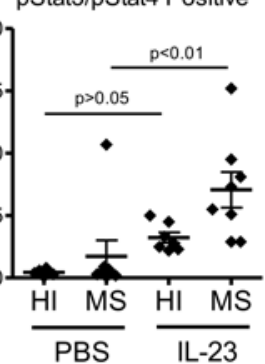

C

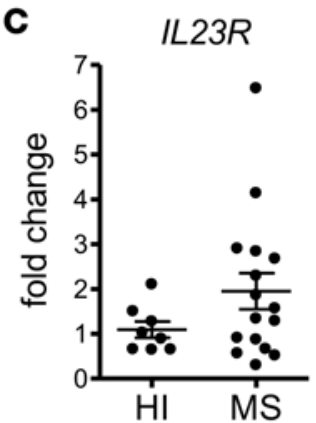

D
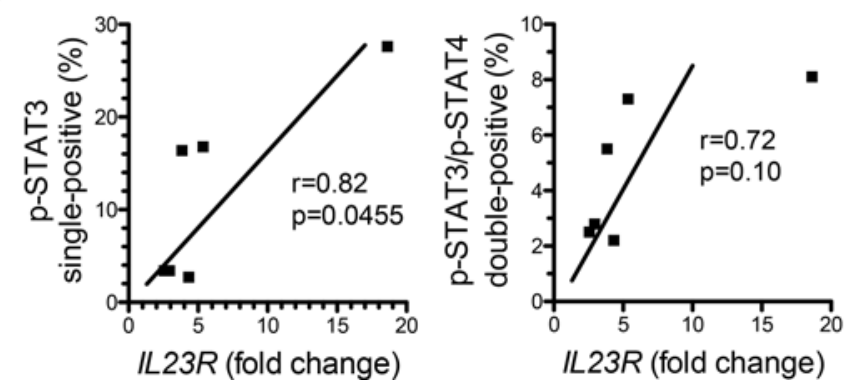

Figure 7. IL-23 induces higher phosphorylation levels of STAT3 and STAT4 in memory CD4+ $T$ cells from MS patients. PBMCs from healthy individuals ( $n=7$ ) and treatment-naive MS $(n=8)$ patients were activated in vitro with anti-CD3 for 2 days. The cells were washed and rested in fresh medium for 4 hours and then were stimulated with either IL-23 or PBS for 20 minutes. Phosphorylation of STAT3 and STAT4 in memory CD4+ ${ }^{+}$cells was analyzed by flow cytometry. Memory $C D 4^{+} T$ cells were gated on $\mathrm{CD}^{+} \mathrm{CD}^{-} \mathrm{CD} 45 \mathrm{RA}^{-}$populations. (A) Three representative samples from healthy individuals and MS patients are shown. $\mathrm{HI}$, healthy individuals. (B) The percentage of p-STAT4 single-positive, p-STAT3 single-positive, and p-STAT3/p-STAT4 double-positive subpopulations in memory $\mathrm{CD} 4^{+} \mathrm{T}$ cells from healthy individuals and MS patients are shown. ${ }^{*} P<0.05$, ${ }^{* *} P<0.01$ (1-way ANOVA with Bonferroni's multiple comparison test). (C) Memory $\mathrm{CD}^{+}$T cells were purified from PBMCs of healthy individuals $(n=8)$ and treatment-naive MS patients $(n=16)$. The purified cells were stimulated with PMA/ ionomycin for 4 hours. IL23R and HPRT mRNA were detected by real-time PCR. Results were normalized with HPRT. Fold change was calculated relative to the average of healthy individuals group (mean \pm SEM) using unpaired Student's $t$ test. (D) The degree of relatedness between the level of $I L 23 R$ expression and percentage of $\mathrm{p}-\mathrm{STAT3}^{+}$or $\mathrm{p}-\mathrm{STAT3}{ }^{+} / \mathrm{p}-\mathrm{STAT4} 4^{+}$cells of the same donor $(n=6)$ was analyzed by nonparametric Pearson correlation test.

p-STAT4 in memory CD4 $4^{+} \mathrm{T}$ cells were detected by gating on memory CD3 ${ }^{+} \mathrm{CD} 8^{-} \mathrm{CD} 45 \mathrm{RA}^{-} \mathrm{T}$ cells (Figure 7A). In the group that had a secondary stimulation with PBS, memory T cells from both MS patients and healthy individuals had minimal p-STAT4 single-positive populations and p-STAT3/p-STAT4 double-positive populations. There were higher p-STAT3 single-positive populations in the cells from MS patients than healthy individuals, but this was not statistically significant (Figure 7B). IL-23 was not efficient in increasing the p-STAT4 single-positive or p-STAT3 single-positive populations in both healthy individuals and MS patients. In contrast, a significant increase of p-STAT3/p-STAT4 double-positive population was observed in the cells from the MS patients upon IL-23 stimulation but not the healthy individuals. The subpopulations of p-STAT3 single-positive and p-STAT3/p-STAT4 double-positive were significantly greater in MS patients than healthy individuals when stimulated with IL-23, whereas the p-STAT4 single-positive population was slightly but not significantly greater in MS patients than healthy individuals 


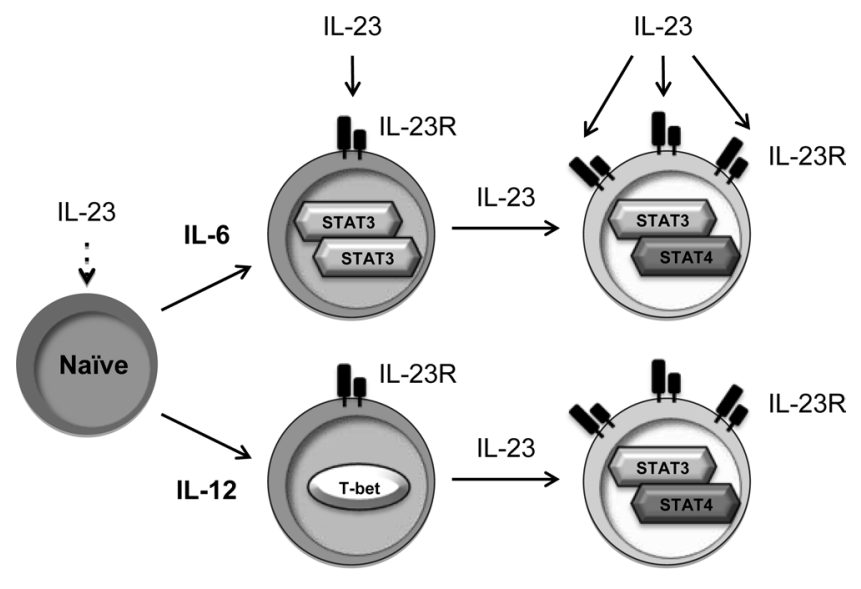

Encephalitogenic
Figure 8. Model of IL-6- and IL-12-induced encephalitogenic T cells. Naive CD4+ $T$ cells, which do not express IL-23R, fail to respond to IL-23. Both IL-6 and IL-12 induce the initial expression of IL-23R. IL-23 signaling further promotes the expression of IL-23R and consequently enhances the sensitivity of IL-23 on those cells. IL-23 signals via p-STAT3/p-STAT4 heterodimers, which might regulate genes contributing to the encephalitogenicity.

(Figure 7B). It was noteworthy that memory T cells from MS patients tended to have higher p-STAT3 levels when compared with healthy individuals in the presence and absence of IL-23. This was consistent with the study reported by Schneider et al., showing that $\mathrm{T}$ cells from MS patients had enhanced p-STAT3 activities (50). These data showed that IL-23 induced coexpression of p-STAT3 and p-STAT4 in human memory $\mathrm{T}$ cells and that memory $\mathrm{T}$ cells from MS patients have higher sensitivity to IL-23 compared with those from healthy individuals. Interestingly, the population of p-STAT3/p-STAT4 double-positive cells is more distinct in the activated memory $\mathrm{CD} 4^{+} \mathrm{T}$ cell population (Figure 7A) compared with the primary activation of naive $\mathrm{CD} 4^{+} \mathrm{T}$ cells (Figure 6C), which may be due to the fact that IL-23R expression is already present on the memory $\mathrm{T}$ cells and must be induced in the naive $\mathrm{T}$ cells.

To verify whether the hypersensitivity to IL-23 was due to a higher expression level of the receptor on memory T cells in MS patients, analysis of IL-23R levels was performed. Since we could not confirm the specificity of human IL-23R antibodies for flow cytometry, real-time PCR of IL23R expression from purified memory CD4 T cells was utilized. Purified memory CD4 ${ }^{+} \mathrm{T}$ cells from healthy individuals and MS patient PBMCs were stimulated with PMA/ionomycin, and the level of IL23R mRNA was measured. PMA/ionomycin was used to briefly activate the memory T cells such that genes for which the memory cells were programmed to express could be detected without significantly altering gene expression due to prolonged TCR activation. The expression of IL23R had a trend to be higher in MS patients than healthy individuals but was not statistically significant $(P=0.0678)$ (Figure 7C). Correlation analysis showed a significant positive correlation between the level of IL-23R and the percentage of p-STAT3 single-positive cells (Figure 7D). A trend toward positive correlation between IL-23R expression and the percentage of p-STAT3/p-STAT4 double-positive cells was also observed, although it was not statistically significant. These data may partially explain the hypersensitivity to IL-23 in memory $\mathrm{T}$ cell from MS patients.

\section{Discussion}

During $\mathrm{CD}^{+} \mathrm{T}$ cell differentiation, APCs secrete a spectrum of cytokines in the microenvironment, and the ratio among specific cytokines defines the phenotype of effector $\mathrm{T}$ cells. Identification of cytokines important for disease has largely been done by genetic deletion. However, these studies give limited information on the biological function of cytokines under normal conditions and typically allow studying only 1 cytokine at a time. In this study, we identified the cytokines critical for inducing the encephalitogenicity of $\mathrm{CD}^{+} \mathrm{T}$ cells by using an in vitro strategy, which was designed to recapitulate the essential signals from APCs. While no single cytokine was sufficient, the combinations of IL-6+IL-23 or IL-12+IL-23 successfully restored the capability of T cells that were activated with anti-CD3/CD28 to transfer EAE. Neutralizing IL-6 or IL-12 in Ag-driven differentiation significantly reduced the disease severity of mice receiving those $\mathrm{T}$ cells, indicating that both cytokines comprised the critical signals for encephalitogenicity provided by APCs. IL-6+IL-23 and IL-12+IL-23 promoted the encephalitogenicity by inducing high levels of IL-23R expression on T cells. Given the fact that IL-23- and IL-23R-deficient mice are completely resistant to EAE $(21,51)$, IL-23 signaling appears to be an essential factor of EAE, regardless of whether it is mediated by Th1 or Th17 cells. Though the effect IL-23 has on Th1 cells has been underappreciated since the discovery of Th17 cells, it had previously been shown that IL-23R was expressed on activated T cells with a Th1 phenotype (45) and that IL-23 enhanced IFN- $\gamma$ production (44). In addition, it was previously shown that myelin-reactive T cells stimulated with IL-23 produced both IFN- $\gamma$ and IL-17 and induced EAE in an IFN- $\gamma$-dependent, IL-17-independent manner (52). These reports are consistent with our finding that IL-12 induces IL-23R expression on Th1 cells. It has been reported that IL-6 signaling through 
STAT3 upregulates the expression of IL-23R $(41,42)$, but the mechanism by which IL-12 induces IL-23R expression needs to be further investigated. We have previously shown that T-bet binds the IL-23 receptor promoter and positively regulates the expression of the Il23r gene (53). Thus, IL-12 may indirectly enhance IL-23R expression via the IFN- $\gamma / \mathrm{T}$-bet pathway. Naive myelin-specific CD4 T cells differentiated with anti-CD3/28+IL-12 and, to a lesser extent, anti-CD3/28+IL-6 had some capacity to induce disease. This is probably due to the fact that IL-12 and IL-6 induced IL-23R expression on the cells and, once the cells were transferred into mice, the myelin-specific T cells may have access to IL-23 in vivo, allowing some cells to fully differentiate into encephalitogenic T cells. It is also interesting that GM-CSF was expressed in the myelin-specific $\mathrm{T}$ cells that were both highly encephalitogenic and nonencephalitogenic, suggesting that, although GM-CSF expression by T cells is necessary to be encephalitogenic (36-39), it is not sufficient. Furthermore, the data indicate that IL-23 is inducing a signaling pathway distinct from GM-CSF that is also necessary for the generation of encephalitogenic $\mathrm{T}$ cells.

Although the concentrations of the cytokines used in this study are at lower concentrations than typically used in in vitro assays, they still may be excessive compared with the in vivo microenvironment in which naive $\mathrm{CD}^{+} \mathrm{T}$ cells are typically differentiated. Thus, cytokine titration may be useful in fully determining the relevance of IL-6, IL-12, and IL-23 in determining encephalitogenicity. Although the present study enhances our understanding of the generation of encephalitogenic T cells, it is still unknown what makes a T cell encephalitogenic. The use of microarray has identified molecules that may be critical, and analysis of these molecules individually and in combination should further our understanding of these molecules and pathways essential for rendering a $\mathrm{CD} 4^{+} \mathrm{T}$ cell encephalitogenic. Another avenue that may be insightful is the study of the differences in what happens to the myelin-specific $\mathrm{T}$ cells generated with the different cytokines after they have been transferred into mice. This will help us understand whether the less encephalitogenic $\mathrm{T}$ cells are defective in proliferation, trafficking, cell recruitment, and other Th functions. If $\mathrm{T}$ cells fail to traffic to the CNS, this may indicate that IL-23R signaling is critical to the upregulation of adhesion molecules or chemokines necessary for migration across the blood brain barrier. If $\mathrm{T}$ cells migrate to the CNS but fail to cause disease, this may indicate that IL-23R signaling is critical to effector $\mathrm{T}$ cell function, such as the production of inflammatory mediators that affect microglia and macrophage functions. An ex vivo microarray analysis of the transferred nonencephalitogenic T cells may also be useful for determining the pathways induced by IL-23R signaling.

IL-23 signaling through STAT3 enhances Th17 cell differentiation. STAT3 binds to several Th17-associated genes, including $I l 17, I l 21, I l 23 r$, and Rorc locus $(54,55)$. Studies using Tg mice deficient in IL-23, IL-23R, or STAT3 in T cells suggest that IL-23 signaling is associated with an essential role in the development of EAE beyond simply promoting IL-17 production $(21,25,26,31,51)$. IL-12 signaling through STAT4 determines Th1 differentiation (48). Mice deficient in IL-12 are susceptible to EAE because Th17 cells can drive atypical disease in the absence of Th1-mediated immunity $(18,19)$. Loss of STAT4 results in resistance to EAE (20); thus, the essential role of STAT4 seems to be independent of IL-12 signaling in regulating autoimmunity. It has previously been shown, and we confirmed in this study, that IL-23 activates both STAT3 and STAT4 $(44,45,56,57)$. Compared with the cells from healthy individuals, memory T cells from MS patients tended to have a higher sensitivity to IL-23, resulting in the phosphorylation of STAT3 and STAT4. This analysis was performed on total memory CD4 T cells, not myelin-specific T cells, suggesting that MS patients may inherently be more responsive to IL-23, which suggests that IL-23R may be a susceptibility factor. $I L-23 R$ polymorphism is associated with multiple autoimmune diseases (58-60), but it has not yet shown a significant correlation with MS risk. An alternative hypothesis is that the inflammatory process in MS may enhance IL-23R sensitivity and perhaps contribute to exacerbation rates or disease progression.

It needs to be further investigated whether $\mathrm{T}$ cell encephalitogenicity is associated with IL-23R downstream of STAT3/STAT4 activity. Our findings provide a mechanism linking the observations of complete resistance in EAE in STAT3- or STAT4-deficient models. It has yet to be determined if the heterodimer directly regulates pathogenic genes that result in encephalitogenicity mediated by IL-23 or if both are necessary independently. ChIP of the DNA bound by p-STAT3/p-STAT4 heterodimers compared with p-STAT3 and p-STAT4 only, and identification of the genes regulated by p-STAT3/p-STAT4 heterodimers, may identify a unique phenotype of encephalitogenic T cells.

In this study, we identified that TCR, CD28 signaling, and the combinations of IL- $6+$ IL-23 or IL-12+IL-23 are the minimal signals required from APCs to generate encephalitogenic T cells. As shown in the proposed model (Figure 8), naive T cells do not respond to IL-23 due to a lack of receptor expression. 
When IL-6 or IL-12 is available in the microenvironment, IL-23R is expressed. IL-23 signaling promotes Il23r gene transcription, creating a positive feedback loop enhancing IL-23R expression, particularly in murine CD4 T cells. In naive human CD4 T cells, IL-12 induced higher levels of IL23R gene expression than IL-6. Although the literature has skewed our attention from Th1 to Th17 as the pathogenic T cells in MS, the sum of the literature supports a dominant role of Th1 cells in MS. Th1 cells are more abundant than Th17 cells in the CSF of MS patients (61), and clinical trials found that administration of IFN- $\gamma$ to MS patients exacerbated disease, while administration of anti-IFN- $\gamma$ slowed disease progression $(62,63)$. In addition, a more thorough analysis of additional cytokines that may induce IL-23R expression on human CD4 T cells is needed, since the cytokine conditions that induce Th17 cells in humans may be different. A comprehensive analysis of the genes induced by p-STAT3 and p-STAT4, independently and as heterodimers, should shed light on the critical role of IL-23R signaling in the generation of encephalitogenic T cells and potentially identify novel therapeutic targets.

\section{Methods}

Animals and adoptive transfer of EAE. MBP Ac1-11-specific V $\alpha 2.3 / \mathrm{V} \beta 8.2$ TCR Tg B10.PL mice have been previously described (64). B10.PL mice were purchased from The Jackson Laboratory. All mice were bred and maintained in a specific pathogen-free animal facility at The Ohio State University.

Activated T cells, as described below, were collected and washed twice with PBS. The cell suspension was injected i.p. into 6- to 12-week-old B10.PL mice at $200 \mu 1$ per mouse. The number of cells transferred in each experiment is stated in the figure legends. EAE disease course was scored on a scale of 0-6: 0, no clinical disease; 1, limp tail; 2, moderate hind limb weakness; 3 , severe hind limb weakness; 4, complete hind limb paralysis; 5 , quadriplegia or premoribund state; and 6, death due to EAE.

Splenocyte preparations. Spleens from 6- to 10-week-old mice were crushed in a PBS-filled Petri dish between the frosted edges of 2 standard microscope slides. Splenocyte suspension was passed through a $70-\mu \mathrm{m}$ cell strainer and centrifuged at $300 \mathrm{~g}$ for 8 minutes at $4^{\circ} \mathrm{C}$, followed by hypertonic rbc lysis. Splenocytes were cultured in medium, consisting of RPMI 1640 (Corning) supplemented with 10\% heat-inactivated FBS (Gibco), 1.25\% HEPES (Corning), 1\% nonessential amino acids (Corning), 1\% L-glutamine (Sigma-Aldrich), 1\% penicillin/streptomycin (Pen/Strep; Sigma-Aldrich), 1\% sodium pyruvate (Corning), and 28.6 $\mu \mathrm{M}$ 2-ME (Sigma-Aldrich).

In vitro murine $C D 4^{+} T$ cell culture. Splenocytes from 6- to 10 -week-old V $\alpha 2.3 / \mathrm{V} \beta 8.2 \mathrm{TCR}$ Tg mice were used as a source of MBP-specific CD4 ${ }^{+} \mathrm{T}$ cells. To generate APC/Ag-activated T cells, TCR Tg splenocytes were cultured in 24 -well plates at $2 \times 10^{6}$ cells/well with $6 \times 10^{6}$ cells/well of irradiated B10.PL splenocytes and activated with $10 \mu \mathrm{g} / \mathrm{ml}$ of MBP Ac1-11 peptide (CS Bio) for 3 days. To generate anti-CD3/CD28activated T cells, 24-well plates were precoated with $1 \mu \mathrm{g} / \mathrm{ml}$ of anti-CD3 and anti-CD28 (145-2C11 and 37.51, BD Pharmingen). TCR Tg splenocytes were incubated in T75 flasks for at least 2 hours to deplete adherent cells and then were plated into anti-CD3/CD28-coated plates at $1 \times 10^{6}$ cells/well with $3 \times 10^{6}$ cells/well of adherent cell-depleted feeder cells for 60 hours.

For experiments comparing $\mathrm{T}$ cells differentiated with different cytokines, adherent cell-depleted splenocytes and naive $\mathrm{CD} 4^{+} \mathrm{T}$ cells from TCR Tg mice were used. Naive $\mathrm{CD} 4^{+} \mathrm{T}$ cells were purified with $\mathrm{CD}^{+} \mathrm{CD} 62 \mathrm{~L}^{+} \mathrm{T}$ Cell Isolation Kit II (Miltenyi Biotec) according to the manufacturer's protocol. Samples with purity $\geq 95 \%$ were used in this study. Purified naive T cells were cultured in anti-CD3/CD28-coated 24 -well plates at $0.5 \times 10^{6}$ cells/well with $3.5 \times 10^{6}$ cells/well of adherent cell-depleted feeder cells for 60 hours. Various cytokines were added into the culture as described as following: IL-23 (5 ng/ml); IL-12 (0.5 ng/ml); IL-6 (20 ng/ml); IL-1 $\beta$ (10 ng/ml); hTGF- $\beta 1$ (1 ng/ml); IFN- $\gamma(5 \mathrm{ng} / \mathrm{ml}) ; \mathrm{IL}-18$ (5 ng/ml); and IL-27 (5 ng/ml). All cytokines except IL-6 were obtained from R\&D Systems. IL-6 was obtained from Miltenyi Biotec.

For the experiment neutralizing IL-6 or IL-12, $4 \mu \mathrm{g} / \mathrm{ml}$ of anti-IL-6 (MP5-20F3, BD Pharmingen) or 1 $\mu \mathrm{g} / \mathrm{ml}$ of anti-IL-12 (polyclonal goat IgG, R\&D Systems) were added, along with $10 \mu \mathrm{g} / \mathrm{ml}$ of MBP Ac111 peptide (CS Bio) into the culture.

Transfection with siRNA in vitro. si-NS was purchased from Dharmacon. STAT4 siRNA (si-STAT4) was purchased from Santa Cruz Biotechnology Inc. (Figure 5), and an independent STAT4 siRNA and control siRNA was purchase from Dharmacon (Supplemental Table 5). Adherent cell-depleted TCR Tg splenocytes were plated in 6-well plates $\left(8 \times 10^{6}\right.$ cells/well). Transfection medium contained $250 \mu 1$ serum-free RPMI1640, $5 \mu$ transIT-TKO reagent (Mirus), and $200 \mu \mathrm{M}$ siRNA and was added to each 
well for 18 hours. Cells were washed with PBS and stimulated with MBP Ac1-11 or plate-bound antiCD3/CD28 plus IL-6+IL-23. The transfection efficiency was $>50 \%$, and knock-down efficiency was determined by Western blotting.

Human subjects and sample processing. For the experiments using naive human $\mathrm{CD} 4^{+} \mathrm{T}$ cells, fresh blood of healthy donors $(n=6)$ was obtained from American Red Cross. Exact information on these donors is not known, but they were "healthy," as defined by the American Red Cross and age 18-50 years. For experiments using memory human $\mathrm{CD}^{+} \mathrm{T}$ cells, blood was obtained by leukapheresis from healthy adult individuals ( 5 female and 3 males, age range 22-52 years) or MS patients (10 female and 6 males, age range 33-54 years; 6 relapsing-remitting MS, 5 secondary progressive MS, and 5 primary progressive MS) after informed consent. Patients were in clinical remission and had not undergone immunomodulatory treatments. PBMCs were isolated from blood over a Ficoll gradient (GE Healthcare). For cryopreservation, PBMCs were suspended in freezing medium, containing 50\% RPMI 1640, 40\% heat-inactivated human serum, and 10\% DMSO and stored in liquid nitrogen.

In vitro human $C D 4^{+}$T cell culture. Fresh PBMCs were isolated from blood of healthy donors obtained from American Red Cross (age 18-50 years, both male and female). CD4 ${ }^{+} \mathrm{CD} 45 \mathrm{RA}^{+} \mathrm{T}$ cells were purified using Naive CD4 ${ }^{+} \mathrm{T}$ cell Isolation Kit II (Miltenyi Biotec). Samples with purity $\geq 95 \%$ were used in this study. Naive T cells were plated at $1 \times 10^{6}$ cells/well in 24 -well plates and activated $25 \mu 1$ of Dynabeads Human T-activator CD3/CD28 (Life Technologies) in the presence of human IL-12 (IL-12; $5 \mathrm{ng} / \mathrm{ml})$, hIL-6 (20 ng/ml), and/or hIL-23 (5 ng/ml) (R\&D Systems). The media consisted of RPMI 1640 with 5\% heat-inactivated human serum AB plasma (Sigma-Aldrich), 1\% HEPES, 1\% L- glutamine, and $1 \%$ Pen/Strep.

Memory $C D 4^{+} T$ cells activation and isolation. Cells were retrievals of cryopreserved samples from both healthy controls and MS patients of similar age, sex, and length of cryopresevation. Approximately $70 \%$ viability was observed after thawing and washing cells in PBS. To activate memory CD4 ${ }^{+} \mathrm{T}$ cells, PBMCs were incubated in T25 flasks for at least 2 hours to deplete adherent cells and then plated at $4 \times 10^{6}$ cells/ well in 24-well plates precoated with anti-human CD3 (HIT3a, BD Pharmingen). After 2 days in culture, the cells were collected, washed, and rested in fresh medium for 4 hours. The cells were stimulated with $20 \mathrm{ng} / \mathrm{ml} \mathrm{hIL}-23$ (R\&D Systems) or PBS for 20 minutes and collected for Phosflow analysis. To isolate memory $\mathrm{CD}^{+} \mathrm{T}$ cells from PBMCs, dead cells were firstly removed with Dead Cell Removal Kit (Miltenyi Biotec), and viable cells were sorted by the negative selection with Memory $\mathrm{CD}^{+} \mathrm{T}$ cell Isolation Kit (Miltenyi Biotec). Pure $(\geq 95 \%) \mathrm{CD}^{+} \mathrm{CD} 45 \mathrm{RO}^{+}$samples were used in this study. Memory $\mathrm{T}$ cells were plated at $1 \times 10^{6}$ to $4 \times 10^{6}$ cells/well in 24 -well plates and stimulated for 4 hours with $50 \mathrm{ng} / \mathrm{ml}$ of PMA and $1 \mu \mathrm{g} / \mathrm{ml}$ of ionomycin.

Flow cytometric analysis. Cells were collected, washed, and resuspended in FACS buffer (1\% BSA in PBS) and incubated with Fc blocker (93, BioLegend) for 10 minutes at $4^{\circ} \mathrm{C}$. Cells were stained for cell-surface markers for 30 minutes at $4^{\circ} \mathrm{C}$. After washing twice with FACS buffer, cells were fixed and permeabilized using Cytofix/Cytoperm solution (BD Biosciences) for 20 minutes at $4^{\circ} \mathrm{C}$. Cells were washed twice using Perm/Wash solution (BD Biosciences) and stained for intracellular cytokines with $\mathrm{Ab}$ diluted in Perm/Wash solution for 30 minutes at $4^{\circ} \mathrm{C}$. Cells were washed twice prior to flow cytometric analysis. Pacific Blue anti-CD4 (RM4-5), FITC anti-CD44 (IM7), APC anti-IFN- $\gamma$ (XMG1.2), and PE anti-IL-17A (TC-1118H10) were obtained from BD Pharmingen. APC anti-IL-23R (FAB1686A) was obtained from R\&D systems.

To detect p-STAT3 and p-STAT4 by flow cytometry, cells from healthy individuals and MS patients (or mouse CD4 T cells) were stimulated with IL-23 or PBS. After 20-30 minutes of stimulation, cells were collected and fixed using Lyse/Fix buffer (BD Phosflow) for 12 minutes at $37^{\circ} \mathrm{C}$. The cells were washed with staining buffer (2\% FBS in PBS) and then permeabilized using ice-cold Perm buffer III (BD Phosflow) for 30 minutes at $4^{\circ} \mathrm{C}$. After washing twice with staining buffer, cells were stained for surface markers and p-STAT protein with $\mathrm{Ab}$ diluted in staining buffer for 1 hour at room temperature. Cells were washed twice prior to flow cytometric analysis. The CD4 marker on human T cells was downregulated due to activation; therefore, memory $\mathrm{CD} 4{ }^{+} \mathrm{T}$ cells were gated on the $\mathrm{CD}^{+} \mathrm{CD}{ }^{-} \mathrm{CD} 45 \mathrm{RA}^{-}$population. FITC antiCD8 (HIT8a, BD Pharmingen), PE-Cy7 anti-CD45RA (HI100, BioLegend), V450 anti-CD3 (UCHT1, BD Horizon), PE anti-p-STAT4 (38/p-Stat4, BD Phosflow), and AF647 anti-p-STAT3 (4/P-STAT3, BD Phosflow) were used for staining. Approximately 100,000 cell events were acquired on a FACSCanto II (BD Biosciences) and analyzed using FlowJo software version 10.1 (Tree Star Inc.). 
ELISA. Supernatants from each cultured condition were collected and analyzed for cytokine production. Sandwich ELISA was performed using purified capture $\mathrm{Ab}$ and biotinylated detection $\mathrm{Ab}$ to measure IFN- $\gamma$ (capture: R46A2; detection: XMG1.2, BD Pharmingen), IL-6 (capture: MP5-20F3; detection: MP532C11, BD Pharmingen), GM-CSF (capture: MP122E9; detection: polyclonal goat IgG, R\&D Systems), and IL-17A (capture: eBio17CK15A5; detection: eBio17B7, eBioscience). Avidin-peroxidase was used to label detection $\mathrm{Ab}$, and TMB Chromogen Solution (Life Technologies) was used as the substrate. The reaction was stopped by adding $2 \mathrm{~N} \mathrm{H}_{2} \mathrm{SO}_{4}$, and $\mathrm{OD}_{450 \mathrm{~nm}}$ was measured on a plate reader (Molecular Devices). Cytokine concentrations were calculated by generating a standard curve using recombinant proteins $(R \& D$ Systems) and analyzed using SoftMax Pro Software version 5.4.5.

Western blot. Whole cell lysate was extracted using M-PER Mammalian Protein Extraction Reagent (Thermo Fisher Scientific) with freshly added proteinase inhibitor cocktail and phosphatase inhibitor cocktail 2 and 3 (Sigma-Aldrich). Samples were prepared by mixing extracted protein with Laemmli sample buffer (Bio-Rad) and heated at $95^{\circ} \mathrm{C}$ for 5 minutes. Samples were electrophoretically separated on $7.5 \%$ Criterion TGX gel (Bio-Rad), transferred to PVDF membranes (Amershan), and then probed with the primary $\mathrm{Ab}$ of interest. Detection was performed using mouse or rabbit HRP-conjugated secondary $\mathrm{Ab}$ (Amershan; Cell Signaling Technology) and followed by developing with SuperSignal West Femto or Pico Substrate (Thermo Fisher Scientific). Films were digitally scanned for protein quantification using the NIH ImageJ software. Ratio of phosphorylation was calculated by comparing the band intensities of p-STAT protein to total STAT protein. STAT3 (124H6), p-STAT3 (M9C6), and $\alpha$-tubulin (DM1A) Ab were purchased from Cell Signaling Technology. STAT4 Ab (C-20) was purchased from Santa Cruz Biotechnology Inc. p-STAT4 Ab (38/p-Stat4) was purchased from BD Biosciences. $\beta$-Actin Ab (AC-74) was purchased from Sigma-Aldrich.

Co-IP. Purified naive murine or human $\mathrm{CD}^{+} \mathrm{T}$ cells were activated with Ag/APC or anti-CD3/28. Nuclear protein was extracted with Pierce NE-PER Nuclear and Cytoplasmic Extraction Reagents (Thermo Fisher Scientific) according to the manufacturer's protocol and was used for co-IP using the same number of cells. STAT3 Ab (124H6, Cell Signaling Technology) was added into each sample at a ratio of 1:200 and incubated at $4^{\circ} \mathrm{C}$ overnight. Protein G Dynabeads (Life Technologies) were incubated with immunocomplex solutions for 30 minutes at $4^{\circ} \mathrm{C}$. The beads were pelleted in a magnetic separation rack and washed 5 times with cold PBS. Beads were resuspended in Laemmli sample buffer and heated at $95^{\circ} \mathrm{C}$ for 5 minutes. The level of p-STAT4 in pull-down immnunocomplexes was determined by Western blot. The blots were also probed for p-STAT3 (D3A7, Cell Signaling Technology) and STAT3 (124H6, Cell Signaling Technology).

$R N A$ extraction and real-time PCR. RNA was isolated with TRIzol Reagent (Ambion), and cDNA was generated with $1 \mu \mathrm{g}$ of RNA/reaction using SuperScript III Reverse Transcriptase (Invitrogen) according to the manufacturer's protocol. RT-PCR was performed using Taqman Universal PCR Master Mix (No AmpErase UNG) and Taqman probes (mouse: Hprt: Mm 015453991_m1 and Il23r. Mm00519943_m1, Tbx21: Mm00450960_m1, Rorc: Mm01261022_m1, Ccr7: Mm00432608_m1, Cd40lg: Mm00441911_m1, Cxcr3: Mm00438259_m1, Il2ra: Mm01340213_m1 and Il7r: Mm01309416_m1; human: HPRT: Hs02800695_ m1, IL23R: Hs00332759_m1, TBX21: Hs00894392_m1, RORC: Hs01076112_m1, IFNG: Hs00989291_m1, IL17A: Hs00174383_m1, CCR7: Hs01013469_m1, CD40LG: Hs00163934_m1, CXCR3: Hs00171041_m1, IL2RA: Hs00907777_m1, IL7R: Hs00902332_m1, CSF2: Hs00929873_m1, CD27: Hs00386811_m1 and IL10: Hs00961622_m1) on a Vii 7 Real-time PCR system (Applied Biosystems). Results were analyzed with the comparative threshold cycle $(\Delta \Delta \mathrm{Ct})$ method and normalized with internal control gene, HPRT. Relative fold change was calculated relative to the average control condition.

${ }^{3} \mathrm{H}$-Thymidine incorporation assay. Splenocytes from TCR Tg mice were cultured in 96-well plates coated with anti-CD3/CD28 at 25,000 cells/well with feeder cells (125,000 cells/well) in various culture conditions for 48 hours. Cells were then pulsed with $30 \mu 1{ }^{3} \mathrm{H}$-Thymidine for 18 hours. Cells were then harvested with the FilterMate Harvester (PerkinElmer) onto Unifilter plates and dried. Microscint20 (30 $\mu 1$; PerkinElmer) was added to each well, and ${ }^{3} \mathrm{H}$-Thymidine incorporation was read using a TopCount NXT machine (PerkinElmer).

Microarray analysis. Splenocytes from naive myelin-specific TCR Tg mice were activated with anti-CD3/ CD28 in the presence of singular or combinations of IL-23, IL-6, and IL-12. At 48 hours after activation, $\mathrm{CD}^{+} \mathrm{T}$ cells were purified using MACS separators with the mouse $\mathrm{CD} 4^{+} \mathrm{T}$ cell Isolation Kit (Miltenyi Biotec), according to the manufacturer's protocol. Purified $\mathrm{CD}^{+} \mathrm{T}$ cells were lysed in TRIzol Reagent, 
followed by RNA isolation. The RNA was further purified with an RNeasy Mini Kit (Qiagen), and RNA integrity/purity was verified. The RNA was reverse transcribed into cDNA and labeled for analysis, using the GeneChip Mouse Genome 4302.0 array (Affymetrix). The microarray analyses were performed in The Genomics Shared Resource, The Ohio State University Comprehensive Cancer Center.

Statistics. The Mann-Whitney $U$ test was used for clinical EAE experiments. Two-tailed unpaired Student $t$ test was used for single comparisons, except for the human data in Figure 6B, in which the samples were paired and a paired Student $t$ test was used. One-way ANOVA with Bonferroni's multiple comparison test was used for multiple comparisons. Two-tailed nonparametric Pearson test was used for correlation analysis. $P<0.05$ was considered significant. Prism software (GraphPad) was used for all statistical analyses.

Study approval. The Ohio State University IRB and the IACUC approved the protocols for this study. In the case of human studies, this includes a statement indicating that written informed consent was received from participants prior to inclusion in the study. However, samples acquired from the American Red Cross were purchased and, thus, do not require OSU IRB approval.

\section{Author contributions}

PWL designed and performed experiments, analyzed data, and wrote the manuscript. A.J. Smith designed and performed experiments, and analyzed data. YY designed experiments and edited manuscript. A.J. Selhorst and YL performed experiments. MKR provided patient samples and edited the manuscript. AELR designed experiments, analyzed data, and assisted with manuscript preparation.

\section{Acknowledgments}

This work was support by grants to AELR from the NIH (1 R01 NS067441) and National Multiple Sclerosis Society (RG 3812). PWL and A.J. Smith were supported by award TL1TR000091 from Clinical Translational Science Award, funded by NIH.

Address correspondence to: Amy E. Lovett-Racke, 460 West $12^{\text {th }}$ Avenue, Room 684, Columbus, Ohio 43065, USA. Phone: 614.688.5647; Email: amy.lovett-racke@osumc.edu.

1. Frohman EM, Racke MK, Raine CS. Multiple sclerosis — the plaque and its pathogenesis. N Engl J Med. 2006;354(9):942-955.

2. Allegretta M, Nicklas JA, Sriram S, Albertini RJ. T cells responsive to myelin basic protein in patients with multiple sclerosis. Science. 1990;247(4943):718-721.

3. Lovett-Racke AE, Trotter JL, Lauber J, Perrin PJ, June CH, Racke MK. Decreased dependence of myelin basic protein-reactive T cells on CD28-mediated costimulation in multiple sclerosis patients. A marker of activated/memory T cells. J Clin Invest. 1998;101(4):725-730

4. Burns J, Bartholomew B, Lobo S. Isolation of myelin basic protein-specific T cells predominantly from the memory T-cell compartment in multiple sclerosis. Ann Neurol. 1999;45(1):33-39.

5. Lovett-Racke AE, Yang Y, Racke MK. Th1 versus Th17: are T cell cytokines relevant in multiple sclerosis? Biochim Biophys Acta. 2011;1812(2):246-251

6. McDonald AH, Swanborg RH. Antigen-specific inhibition of immune interferon production by suppressor cells of autoimmune encephalomyelitis. J Immunol. 1988;140(4):1132-1138.

7. Ando DG, Clayton J, Kono D, Urban JL, Sercarz EE. Encephalitogenic T cells in the B10.PL model of experimental allergic encephalomyelitis (EAE) are of the Th-1 lymphokine subtype. Cell Immunol. 1989;124(1):132-143.

8. Waldburger KE, Hastings RC, Schaub RG, Goldman SJ, Leonard JP. Adoptive transfer of experimental allergic encephalomyelitis after in vitro treatment with recombinant murine interleukin-12. Preferential expansion of interferon-gamma-producing cells and increased expression of macrophage-associated inducible nitric oxide synthase as immunomodulatory mechanisms. Am J Pathol. 1996;148(2):375-382.

9. Yura M et al. Role of MOG-stimulated Th1 type "light up" (GFP+) CD4+ T cells for the development of experimental autoimmune encephalomyelitis (EAE). J. Autoimmun. 2001;17(1):17-25.

10. Olsson T, et al. Autoreactive T lymphocytes in multiple sclerosis determined by antigen-induced secretion of interferon- $\gamma$. J Clin Invest. 1990;86(3):981-985.

11. Sun JB, et al. Autoreactive T and B cells responding to myelin proteolipid protein in multiple sclerosis and controls. Eur J Immunol. 1991;21(6):1461-1468.

12. Voskuhl RR, Martin R, Bergman C, Dalal M, Ruddle NH, McFarland HF. T helper 1 (Th1) functional phenotype of human myelin basic protein-specific T lymphocytes. Autoimmunity. 1993;15(2):137-143.

13. Pelfrey CM, Rudick RA, Cotleur AC, Lee JC, Tary-Lehmann M, Lehmann PV. Quantification of self-recognition in multiple sclerosis by single-cell analysis of cytokine production. J Immunol. 2000;165(3):1641-1651.

14. Lublin FD, et al. Monoclonal anti- $\gamma$ interferon antibodies enhance experimental allergic encephalomyelitis. Autoimmunity. $1993 ; 16(4): 267-274$ 
15. Heremans H, Dillen C, Groenen M, Martens E, Billiau A. Chronic relapsing experimental autoimmune encephalomyelitis (CREAE) in mice: enhancement by monoclonal antibodies against interferon-gamma. Eur J Immunol. 1996;26(10):2393-2398.

16. Ferber IA, et al. Mice with a disrupted IFN- $\gamma$ gene are susceptible to the induction of experimental autoimmune encephalomyelitis (EAE). J Immunol. 1996;156(1):5-7.

17. Willenborg DO, Fordham S, Bernard CC, Cowden WB, Ramshaw IA. IFN- $\gamma$ plays a critical down-regulatory role in the induction and effector phase of myelin oligodendrocyte glycoprotein-induced autoimmune encephalomyelitis. J Immunol. 1996;157(8):3223-3227.

18. Becher B, Durell BG, Noelle RJ. Experimental autoimmune encephalitis and inflammation in the absence of interleukin-12. J Clin Invest. 2002;110(4):493-497.

19. Gran B, et al. IL-12p35-deficient mice are susceptible to experimental autoimmune encephalomyelitis: evidence for redundancy in the IL-12 system in the induction of central nervous system autoimmune demyelination. J Immunol. 2002;169(12):7104-7110.

20. Chitnis T, et al. Effect of targeted disruption of STAT4 and STAT6 on the induction of experimental autoimmune encephalomyelitis. J Clin Invest. 2001;108(5):739-747.

21. Cua DJ, et al. Interleukin-23 rather than interleukin-12 is the critical cytokine for autoimmune inflammation of the brain. Nature. 2003;421(6924):744-748.

22. Langrish CL, et al. IL-23 drives a pathogenic T cell population that induces autoimmune inflammation. J Exp Med. 2005;201(2):233-240.

23. Lock C, et al. Gene-microarray analysis of multiple sclerosis lesions yields new targets validated in autoimmune encephalomyelitis. Nat Med. 2002;8(5):500-508.

24. Tzartos JS, et al. Interleukin-17 production in central nervous system-infiltrating $\mathrm{T}$ cells and glial cells is associated with active disease in multiple sclerosis. Am J Pathol. 2008;172(1):146-155.

25. Komiyama Y, et al. IL-17 plays an important role in the development of experimental autoimmune encephalomyelitis. J Immunol. 2006;177(1):566-573.

26. Haak S, et al. IL-17A and IL-17F do not contribute vitally to autoimmune neuro-inflammation in mice. J Clin Invest. 2009;119(1):61-69.

27. Veldhoen M, Hocking RJ, Atkins CJ, Locksley RM, Stockinger B. TGF- $\beta$ in the context of an inflammatory cytokine milieu supports de novo differentiation of IL-17-producing T cells. Immunity. 2006;24(2):179-189.

28. Bettelli E, et al. Reciprocal developmental pathways for the generation of pathogenic effector TH17 and regulatory T cells Nature. 2006;441(7090):235-238

29. Acosta-Rodriguez EV, Napolitani G, Lanzavecchia A, Sallusto F. Interleukins $1 \beta$ and 6 but not transforming growth factor- $\beta$ are essential for the differentiation of interleukin 17-producing human T helper cells. Nat Immunol. 2007;8(9):942-949.

30. Samoilova EB, Horton JL, Hilliard B, Liu TS, Chen Y. IL-6-deficient mice are resistant to experimental autoimmune encephalomyelitis: roles of IL-6 in the activation and differentiation of autoreactive T cells. J Immunol. 1998;161(12):6480-6486

31. Harris TJ, et al. Cutting edge: An in vivo requirement for STAT3 signaling in TH17 development and TH17-dependent autoimmunity. J Immunol. 2007;179(7):4313-4317.

32. McGeachy MJ, et al. TGF- $\beta$ and IL- 6 drive the production of IL-17 and IL-10 by T cells and restrain T(H)-17 cell-mediated pathology. Nat Immunol. 2007;8(12):1390-1397.

33. Yang Y, et al. T-bet is essential for encephalitogenicity of both Th1 and Th17 cells. J Exp Med. 2009;206(7):1549-1564

34. Ghoreschi K, et al. Generation of pathogenic T(H)17 cells in the absence of TGF- $\beta$ signalling. Nature. 2010;467(7318):967-971.

35. Lee PW, Yang Y, Racke MK, Lovett-Racke AE. Analysis of TGF- $\beta 1$ and TGF- $\beta 3$ as regulators of encephalitogenic Th17 cells: Implications for multiple sclerosis. Brain Behav Immun. 2015;46:44-49.

36. McQualter JL, et al. Granulocyte macrophage colony-stimulating factor: a new putative therapeutic target in multiple sclerosis J Exp Med. 2001;194(7):873-882.

37. Ponomarev ED, Shriver LP, Maresz K, Pedras-Vasconcelos J, Verthelyi D, Dittel BN. GM-CSF production by autoreactive T cells is required for the activation of microglial cells and the onset of experimental autoimmune encephalomyelitis. $J$ Immunol. 2007; 178(1):39-48.

38. Codarri L, et al. ROR $\gamma \mathrm{t}$ drives production of the cytokine GM-CSF in helper T cells, which is essential for the effector phase of autoimmune neuroinflammation. Nat Immunol. 2011;12(6):560-567.

39. El-Behi M, et al. The encephalitogenicity of T(H)17 cells is dependent on IL-1- and IL-23-induced production of the cytokine GM-CSF. Nat Immunol. 2011;12(6):568-575.

40. Awasthi A, et al. Cutting edge: IL-23 receptor gfp reporter mice reveal distinct populations of IL-17-producing cells. J Immunol. 2009;182(10):5904-5908.

41. Yang XO, et al. STAT3 regulates cytokine-mediated generation of inflammatory helper T cells. J Biol Chem 2007;282(13):9358-9363.

42. Zhou L et al. IL-6 programs TH-17 cell differentiation by promoting sequential engagement of the IL-21 and IL-23 pathways. Nat. Immunol. 2007;8(9):967-974.

43. Aggarwal S, Ghilardi N, Xie MH, de Sauvage FJ, Gurney AL. Interleukin-23 promotes a distinct CD4 T cell activation state characterized by the production of interleukin-17. J Biol Chem. 2003;278(3):1910-1914

44. Oppmann B, et al. Novel p19 protein engages IL-12p40 to form a cytokine, IL-23, with biological activities similar as well as distinct from IL-12. Immunity. 2000;13(5):715-725.

45. Parham C et al. A receptor for the heterodimeric cytokine IL-23 is composed of IL-12R 1 and a novel cytokine receptor subunit, IL-23R. J Immunol. 2002;168(11):5699-5708.

46. Lee YK, et al. Late developmental plasticity in the T helper 17 lineage. Immunity. 2009;30(1):92-107.

47. Mathur AN, et al. Stat3 and Stat4 direct development of IL-17-secreting Th cells. J Immunol. 2007;178(8):4901-4907.

48. Thierfelder WE, et al. Requirement for Stat4 in interleukin-12-mediated responses of natural killer and T cells. Nature 1996;382(6587):171-174.

49. Becher B, Durell BG, Noelle RJ. Experimental autoimmune encephalitis and inflammation in the absence of interleukin-12. J Clin Invest. 2002;110(4):493-497. 
50. Schneider A, et al. In active relapsing-remitting multiple sclerosis, effector T cell resistance to adaptive T(regs) involves IL-6-mediated signaling. Sci Transl Med. 2013;5(170):170ra15.

51. Zhang GX, et al. Role of IL-12 receptor $\beta 1$ in regulation of T cell response by APC in experimental autoimmune encephalomyelitis. J Immunol. 2003;171(9):4485-4492.

52. Kroenke MA, Segal BM. IL-23 modulated myelin-specific T cells induce EAE via an IFN $\gamma$ driven, IL-17 independent pathway. Brain Behav Immun. 2011;25(5):932-937.

53. Gocke AR, et al. T-bet regulates the fate of Th1 and Th17 lymphocytes in autoimmunity. J Immunol. 2007;178(3):1341-1348.

54. Durant L, et al. Diverse targets of the transcription factor STAT3 contribute to T cell pathogenicity and homeostasis. Immunity. 2010;32(5):605-615

55. Yang XP, et al. Opposing regulation of the locus encoding IL-17 through direct, reciprocal actions of STAT3 and STAT5. Nat Immunol. 2011;12(3):247-254

56. Lankford CS, Frucht DM. A unique role for IL-23 in promoting cellular immunity. J Leukoc Biol. 2003;73(1):49-56.

57. Watford WT, Hissong BD, Bream JH, Kanno Y, Muul L, O'Shea JJ. Signaling by IL-12 and IL-23 and the immunoregulatory roles of STAT4. Immunol Rev. 2004;202:139-156

58. Duerr RH, et al. A genome-wide association study identifies IL23R as an inflammatory bowel disease gene. Science. 2006;314(5804):1461-1463.

59. Beaudoin M, et al. Deep resequencing of GWAS loci identifies rare variants in CARD9, IL23R and RNF186 that are associated with ulcerative colitis. PLoS Genet. 2013;9(9):e1003723.

60. Kirino Y, et al. Genome-wide association analysis identifies new susceptibility loci for Behçet's disease and epistasis between HLA-B*51 and ERAP1. Nat Genet. 2013;45(2):202-207.

61. Brucklacher-Waldert V, Stuerner K, Kolster M, Wolthausen J, Tolosa E. Phenotypical and functional characterization of T help er 17 cells in multiple sclerosis. Brain. 2009;132(pt 12):3329-3341.

62. Panitch HS, Hirsch RL, Haley AS, Johnson KP. Exacerbations of multiple sclerosis in patients treated with $\gamma$ interferon. Lancet. 1987;1(8538):893-895.

63. Skurkovich S, et al. Randomized study of anitbodies to IFN- $\gamma$ and TNF- $\alpha$ in secondary progressive multiple sclerosis. Mult. Scler. 2001;7(5):277-284.

64. Goverman J, et al. Transgenic mice that express a myelin basic protein-specific $\mathrm{T}$ cell receptor develop spontaneous autoimmunity. Cell. 1993;72(4):551-560. 